\title{
School choice with asymmetric information: Priority design and the curse of acceptance
}

\author{
Andrew Kloosterman \\ Department of Economics, University of Virginia \\ Peter Troyan \\ Department of Economics, University of Virginia
}

\begin{abstract}
We generalize standard school choice models to allow for interdependent preferences and differentially informed students. We show that, in general, the commonly used deferred acceptance mechanism is no longer strategy-proof, the outcome is not stable, and may make less informed students worse off. We attribute these results to a curse of acceptance. However, we also show that if priorities are designed appropriately, positive results are recovered: equilibrium strategies are simple, the outcome is stable, and less informed students are protected from the curse of acceptance. Our results have implications for the current debate over priority design in school choice.
\end{abstract}

KeYwords. Matching, stability, asymmetric information.

JEL CLASSIFICATION. C78, D47.

\section{Introduction}

Please accept my resignation. I don't want to belong to any club that would have me as a member. Groucho Marx

In the last decade, school choice has rapidly expanded across the United States and around the world, which has led to a vast and rapidly expanding economics literature, encompassing a wide array of theoretical, practical, and empirical papers. On the mechanism design side of this literature, the standard modeling approach emanates from the seminal paper of Abdulkadiroğlu and Sönmez (2003), and is the analogue of what the broader mechanism design/auctions literature refers to as private values: each student is perfectly informed about her own ordinal preference relation over all schools and is asked to report these preferences to a mechanism, which determines the assignment. While this simplifies the analysis, it abstracts away from differentially informed students who likely exist in practice. For example, if some parents have less time or resources to

Andrew Kloosterman: ask5b@virginia.edu

Peter Troyan: troyan@virginia.edu

Authors names appear in rnadom order. We would like to thank (also in random order) Marek Pycia, Al Roth, Assaf Romm, and Fuhito Kojima for helpful comments, as well as the audiences at the University of Zurich, the 2017 INFORMS Annual Meeting, the 14th Matching in Practice Workshop in Cologne, and the 2018 SAET Conference in Taipei. We gratefully acknowledge the Bankard Fund for Political Economy for supporting this research. Troyan acknowledges support from the Roger Sherman Fellowship.

(C) 2020 The Authors. Licensed under the Creative Commons Attribution-NonCommercial License 4.0. Available at https://econtheory.org. https://doi.org/10.3982/TE3621 
research and visit schools to learn about their quality, they may be less informed than others, as captured by the following excerpt ("Not Everyone Has a Choice," US News \& World Report, August 31, 2015):

School choice policies are predicated on the assumption that parents have enough information to make an informed decision on where to send their children. However, the vast majority of school districts do not provide families with robust school quality information.... Research also shows that districts don't always do a good job of disseminating information and explaining options to certain groups-low-income parents and those whose children are first-generation Americans, for example.

In such situations, a closer theoretical analogue might be the other canonical model routinely studied in auctions and mechanism design, i.e., common values: every agent has the same value, but agents may have different information about what this value is. This manifests in school choice if all parents care about a school's quality, but some are more informed about which schools are higher quality than others. More generally, quality might be one of several factors that influence student preferences (other examples include location, sibling attendance, etc.), and so student preferences are likely correlated, although perhaps not perfectly so. The goal of this paper is to reexamine school choice in a more realistic, interdependent values framework.

Perhaps the most well known result from the literature on auctions with interdependent values is the so-called winner's curse. We start by identifying a related "curse" in our environment, which we call the curse of acceptance: upon observing their assignment, less informed students update their belief about the quality of their assigned school downward. Intuitively, the more informed students submit preferences with high quality schools at the top of their lists, leaving more empty seats at the low quality schools for the less informed students. While the less informed may not know which schools are high quality or low quality, they are at least aware that there are others who do have such information, and so they can infer that any school to which they are eventually assigned is likely to be of lower quality on average. In short, just like Groucho, less informed students would prefer not to enroll at the school that admits them.

This environment leads to the failure of many standard properties of well known assignment mechanisms, such as strategy-proofness and stability. First, strategyproofness as conventionally understood is not feasible (for any mechanism), as some students do not know their true preferences. In fact, as we show, determining an optimal strategy can be very complex for the less informed students, and dominant strategies generally do not exist. Second, deferred acceptance (DA), the standard stable mechanism in the usual model and the most popular mechanism in applications, may no longer produce a stable match. Indeed, the standard definition of stability no longer applies when students may not know their preferences. We thus introduce a definition of stability for our environment that requires that no student's expected utility from successfully rematching, conditional on her information, is higher than her expected utility from remaining at her assigned school. We show that stability may not hold in general, and attribute this to the curse of acceptance: less informed students are able to infer that other schools are likely better than their assignment, and they may have high enough priority at one of these schools to block the original match. This is important, because 
instability is not just a theoretical concern. For instance, in New York City in 2004, over $5 T 000$ students appealed the assignment they received from the (supposedly stable) DA mechanism, with 300 of these appeals coming from students who received their stated first choice (Abdulkadiroğlu et al. 2005).

Our negative results rely on showing that desirable properties fail for some specific priority structure. In the second half of the paper, we show that these negative results motivate looking at the problem in a new light, as one of priority design. We show that if priorities are designed in an appropriate way, positive results are recovered. In particular, we introduce the idea of a secure school, which is a school $s$ such that a student $j$ 's priority ranking at $s$ is below the capacity of $s$. In other words, a secure school is a default option that $j$ can be certain she will receive, as long as she ranks it first. We consider what happens when the priority structure is designed so that each student has a secure school in two models: perfectly correlated preferences in the standard finite economy model and imperfectly correlated preferences in a model with a continuum of students. In both cases, we show that stable matchings exist and the DA mechanism finds one. Additionally, while DA is still not strategy-proof, the equilibrium strategies are simple and focal.

Last, and perhaps most importantly, our informational environment highlights another new issue not found in the earlier school choice literature relating to the welfare consequences of school choice. An important goal of school choice is to provide all students (and in particular, those in poorer neighborhoods) with a fair chance of attending a good school. However, if students are differentially informed, the informed students will, on average, be assigned to better schools at the expense of the less informed, who may be worse off. The US News \& World Report quote suggests that it is likely the case that those in poorer neighborhoods are these less informed students and so school choice could exacerbate the issue of them not receiving a fair chance of attending a good school. Indeed, in our continuum model, introducing school choice is actually a Pareto disimprovement for the less informed students when there are no secure schools: because of the curse of acceptance, less informed students may actually be worse off than if there were no school choice at all. However, when all students have a secure school, this is no longer true, and introducing school choice is a Pareto improvement. In particular, we show that choice is valuable, as the informed students can use their information to improve their own assignment, but they do not do this simply at the expense of the uninformed students. Thus, the use of secure schools can be seen as a practical compromise: it allows for choice for students who know their preferences, which increases efficiency, while still protecting the less informed students from the unintended consequences of the curse of acceptance.

Related literature The earliest paper to look at incomplete information in a matching environment is Roth (1989). He considers a model where everyone knows their own preferences, but have incomplete information about the preferences of others, while in our model, students may not know even their own preferences. In a similar vein as Roth (1989), Ehlers and Massó $(2007,2015)$ study ordinal Bayes-Nash equilibria when agents have incomplete information about others' preferences. 
There is other recent work that looks at stability with incomplete information about one's own preferences including Liu et al. (2014), Bikhchandani (2017), and Liu (2019). Liu et al. (2014) consider a model of informed workers and uninformed firms, and introduce an iterative stability concept similar in spirit to the rationalizability ideas of Bernheim (1984) and Pearce (1984). They define blocking in a "belief-free" way: firms will join a blocking pair if they are better off with all types of a worker that are willing to join, and then iteratively remove matching outcomes that are blocked until nothing more is blocked. Bikhchandani (2017) extends the belief-free notion of blocking to a Bayesian notion but follows the same iterative procedure. More closely related is Liu (2019), who presumes firms have a common prior over worker types and studies which mappings from states of the world to realized matchings are stable when agents update their beliefs using Bayes' rule. We follow this approach as well, but Liu takes the mapping as exogenously given, whereas in our model, it is an equilibrium object induced by the school choice mechanism that is used.

Chakraborty et al. (2010) and Chakraborty et al. (2015) (hereafter, CCO) take a "mechanism design" approach to studying matching mechanisms under interdependent values. They also show impossibility results regarding stability under interdependent values, though our formal model and the driving force behind our impossibility results are distinct. ${ }^{1}$ On the positive side, CCO show that a serial dictatorship is stable in their model when one side of the market has a common ranking over the other and this is known. Our positive results allow for a larger class of admissible priority structures, which has important implications for the design of priorities and the welfare of the students, results which are novel to this paper. Finally, there is also a literature on interdependent values in decentralized matching (e.g., Chade (2006), Lee (2009), Chade et al. (2014), Che and Koh (2016)). Most notably, Chade (2006) identifies a phenomenon he calls the "acceptance curse effect" in a dynamic marriage model where men and women randomly meet each period and decide whether to accept their current partner and leave or wait and get a new draw in the next period.

\section{Preliminaries}

\subsection{Model}

Let $J=\left\{j_{1}, \ldots, j_{N}\right\}$ be a set of students and let $S=\left\{s_{1}, \ldots, s_{M}\right\}$ be a set of schools. Each school $s$ has capacity $q_{s}$ and a priority relation $\succ_{s}$, where $\succ_{s}$ is a strict, complete, and transitive binary relation over $J$. We write $q=\left(q_{s}\right)_{s \in S}$ and $\succ=\left(\succ_{s}\right)_{s \in S}$ to denote the capacity vector and priority profile for all schools, respectively. We assume that $\sum_{s \in S} q_{s} \geq N$, which is a standard assumption in school choice where each student must legally be offered a seat at some school.

\footnotetext{
${ }^{1}$ They consider mechanisms where agents report their "signal" to the designer, and the counterexample for their weak stability—which is the closest analogue to our concept of stability-exhibits an informed party who wants to lie about his preferences to mislead an uninformed party into matching with an undesirable agent, while in our model, informed students have a dominant strategy to truthfully reveal, and stability fails due to the curse of acceptance outlined above.
} 
We model preference interdependencies by having the students' preferences depend on an underlying state $\omega$. The state space $\Omega$ is finite, with an associated probability distribution $\operatorname{Pr}: \Omega \rightarrow[0,1]$ such that $\sum_{\omega \in \Omega} \operatorname{Pr}(\omega)=1$. Every student has a (statedependent) utility function, where $u_{j}^{\omega}(s)$ is student $j$ 's utility for school $s$ in state $\omega$. We assume that preferences are strict, i.e., given a state $\omega, u_{j}^{\omega}(s) \neq u_{j}^{\omega}\left(s^{\prime}\right)$ for all $s \neq s^{\prime}$ and denote $j$ 's strict ordinal preferences in state $\omega$ by $P_{j}(\omega)$. Where relevant, agents evaluate lotteries using von Neumann-Morgenstern preferences. In particular, a student's expected utility from matching to school $s$ given information that the true state lies in some subset of the state space $\mathcal{I}^{\prime} \subseteq \Omega$ is written

$$
E\left(u_{j}^{\omega}(s) \mid \mathcal{I}^{\prime}\right)=\sum_{\omega \in \Omega} u_{j}^{\omega}(s) \operatorname{Pr}\left(\omega \mid \mathcal{I}^{\prime}\right),
$$

where $\operatorname{Pr}\left(\omega \mid \mathcal{I}^{\prime}\right)$ is the posterior probability of the true state being $\omega$ conditional on information $\mathcal{I}^{\prime}$ and is obtained via Bayes' rule.

After Nature draws $\omega$, each student receives a signal $\mathcal{I}_{j}(\omega) \subseteq \Omega$, where $\mathcal{I}_{j}(\omega)$ denotes the subset of states that are possible given $j$ 's signal when the true state is $\omega$. We partition the students into a set of informed students, $I$, who receive a signal that allows them to learn their own ordinal preferences, and a set of uninformed students, $U$, who receive a completely uninformative signal. Formally, $J=I \cup U$ and, for all $j \in I$, we have $\mathcal{I}_{j}(\omega)=$ $\left\{\omega^{\prime}: P_{j}\left(\omega^{\prime}\right)=P_{j}(\omega)\right\}$ for all $\omega \in \Omega$, while for all $j \in U$, we have $\mathcal{I}_{j}(\omega)=\Omega$ for all $\omega \in \Omega$.

The state space $\Omega$ defined here is quite flexible, but in what follows we concretely define state spaces where the students' utility for a school is determined by an intrinsic quality, which is common to all students, and, later in the paper, also include an idiosyncratic component that is individual to each student. We also consider only the two levels of information: perfectly informed about own preferences and completely uninformed. While it is also possible with this model to define finer levels of "partial informativeness" on the part of the students, we found that we are able to convey our main points with this simple, parsimonious formulation, where the informed students represent those whose parents exert time and effort to learn quality, and the uninformed students' represent those whose parents do not. Exploring further findings with more realistic partially informed students is an interesting direction for future research, though, given the complexities that arise even in the simpler model we consider, we expect that in such a model, it will be quite challenging to state clear theoretical results.

\subsection{Matchings and mechanisms}

A matching is a function $\mu: J \cup S \rightarrow 2^{J \cup S}$ such that (i) $\mu_{j} \in S$ for all $j \in J$, (ii) $\mu_{s} \subseteq J$ and $\left|\mu_{s}\right| \leq q_{s}$ for all $s \in S$, and (iii) $\mu_{j}=s$ if and only if $j \in \mu_{s}$. In words, $\mu_{j}$ is the school assigned to student $j$ and $\mu_{s}$ is the set of students assigned to school $s$. Let $\mathcal{M}$ denote the set of all possible matchings.

So as to implement a "good" matching, market organizers (e.g., school districts) must elicit the private information of the agents. In most real-world settings, the way this is done is by asking the agents to submit an ordinal preference ranking over the 
set of schools, and then using some mechanism to output a final matching. To be consistent with this, throughout this paper, we define mechanisms in this way; formally, a mechanism is a function $\psi: \mathcal{P}^{N} \rightarrow \mathcal{M}$, where $\mathcal{P}$ is the set of all strict ordinal preference relations over $S$, and $P=\left(P_{j}\right)_{j \in J} \in \mathcal{P}^{N}$ denotes a profile of preference relations, one for each student. Given some $P_{j} \in \mathcal{P}$, we write $s P_{j} s^{\prime}$ to denote that $s$ is strictly preferred to $s^{\prime}$ and write $s R_{j} s^{\prime}$ if either $s P_{j} s^{\prime}$ or $s=s^{\prime}$. We use $\operatorname{rank}_{P_{j}}(s)=\left|\left\{s^{\prime}: s^{\prime} R_{j} s\right\}\right|$ to denote the ranking of $s$ in the preference relation $P_{j}$. We write $\psi_{j}(P)$ to denote student $j$ 's assignment under mechanism $\psi$ when the submitted reports are $P$. Similarly, $\psi_{s}(P)$ denotes the assignment of school $s$. Finally, for shorthand, we sometimes write a preference relation as a string of schools, e.g., $P_{j}=s^{(1)} s^{(2)} \cdots s^{(M)}$, where $s^{(1)}$ is $j$ 's most preferred school, $s^{(2)}$ is the second-most preferred school, etc.

A mechanism $\psi$ induces a game in which the action space for each player is $\mathcal{P}$. A strategy for student $j$ in this game is a mapping $\sigma_{j}: \Omega \rightarrow \mathcal{P}$ that is measurable with respect to her information (i.e., for any two states $\omega$ and $\omega^{\prime}$ where $\mathcal{I}_{j}(\omega)=\mathcal{I}_{j}\left(\omega^{\prime}\right)$, it must be that $\left.\sigma_{j}(\omega)=\sigma_{j}\left(\omega^{\prime}\right)\right)$. A profile of strategies $\sigma=\left(\sigma_{1}, \ldots, \sigma_{N}\right)$ is a (Bayesian) equilibrium of the game induced by mechanism $\psi$ if

$$
E\left[u_{j}^{\omega}\left(\psi_{j}(\sigma(\omega))\right) \mid \mathcal{I}_{j}(\omega)\right] \geq E\left[u_{j}^{\omega}\left(\psi_{j}\left(\sigma_{j}^{\prime}(\omega), \sigma_{-j}(\omega)\right)\right) \mid \mathcal{I}_{j}(\omega)\right]
$$

for all other strategies $\sigma_{j}^{\prime}$, all $j \in J$, and all $\omega \in \Omega$.

In standard matching models, a mechanism is said to be strategy-proof for the students if there does not exist a $j \in J$ and preferences $P_{j}, P_{j}^{\prime}, P_{-j}$ such that $\psi_{j}\left(P_{j}^{\prime}, P_{-j}\right) P_{j} \times$ $\psi_{j}\left(P_{j}, P_{-j}\right)$. In words, reporting true preferences is a dominant strategy of the induced preference revelation game. Strategy-proofness is a desirable property because it makes the mechanism simple for students to play. Strategy-proofness is not actually a feasible property in our model, since uninformed students do not learn their preferences, and reporting them is, therefore, not measurable; however, it should be clear that if a mechanism is strategy-proof in the sense just defined, it will be a dominant strategy for the informed students to report their true preferences.

\subsection{Full matchings and stability}

Suppose the state is $\omega$. In the classical matching literature, given a matching $\mu$, a student and a school $(j, s)$ are called a (classical) blocking pair if (in our notation) (i) $u_{j}^{\omega}(s)>u_{j}^{\omega}\left(\mu_{j}\right)$ and (ii) either $\left|\mu_{s}\right|<q_{s}$ or $j \succ_{s} j^{\prime}$ for some $j^{\prime} \in \mu_{s}$. A matching $\mu$ is then called (classically) stable if there are no classical blocking pairs. ${ }^{2}$ In the school choice literature, stability is sometimes called fairness and is given a normative interpretation

\footnotetext{
${ }^{2}$ In the sequel, we reserve the word "stability" to refer to the concept in the incomplete information sense to be defined below; when we want to refer to the standard definition of stability defined here, we write "classical stability." Also, an additional component of stability usually imposed is that every student prefers her assigned school to being unmatched, and no school wants to unilaterally drop one of its assigned students. We assume that students find all schools acceptable and schools find all students acceptable, so this part of stability is not an issue. We assume this only for ease of exposition; none of our results is driven by this assumption.
} 
in that a stable outcome is desirable because no student will justifiably envy the school assignment of a student over which she has higher priority at that school.

This definition of stability must be generalized to account for incomplete information. To do so, we take a stance on the timing of the mechanism and what students observe at each stage, which we do as follows: students submit preference reports, the mechanism determines a matching, students observe the school they are matched to, students (may) propose blocking with another school, and blocking pairs are formed if proposing students have higher priority than another student at the school or if the school is not at capacity. Note in particular that students observe only their own school, rather than the entire (realized) matching. We remark further on these points below, after introducing the definition of stability formally.

To formally define stability in our environment, we must first introduce the concept of a full matching, which is a mapping from states to observed matchings. This is an important object in our setting, because strategies in the preference revelation game induce this object.

Definition 1. A full matching is a function $\boldsymbol{\mu}: \Omega \rightarrow \mathcal{M}$ that assigns a matching to each state $\omega \in \Omega$.

We use bold face type, $\boldsymbol{\mu}$, to denote full matchings, and write $\boldsymbol{\mu}^{\omega} \in \mathcal{M}$ for the matching in state $\omega$, write $\boldsymbol{\mu}_{j}^{\omega}$ for the assignment of student $j$ in matching $\boldsymbol{\mu}^{\omega}$, and write $\boldsymbol{\mu}_{s}^{\omega}$ for the assignments of school $s$ in matching $\boldsymbol{\mu}^{\omega}$.

A student who is considering proposing a block knows the full matching $\boldsymbol{\mu}$ (an equilibrium object), and so can narrow down the set of states, first, to those that are consistent with the school they have observed they are matched to and, second, to those in which they have priority high enough to successfully form a blocking pair (which includes the possibility of blocking with an empty seat in some state). To capture this formally, we define the following two sets, where, to avoid notational clutter, we suppress their dependence on $\mu$ :

$$
\begin{aligned}
\mathcal{A}_{j}\left(s^{\prime}\right) & =\left\{\omega \in \Omega: \boldsymbol{\mu}_{j}^{\omega}=s^{\prime}\right\}, \\
\mathcal{B}_{j}(s) & =\left\{\omega \in \Omega: \boldsymbol{\mu}_{j}^{\omega} \neq s \text { and }\left|\boldsymbol{\mu}_{s}^{\omega}\right|<q_{s} \text { or } j \succ_{s} j^{\prime} \text { for some } j^{\prime} \in \boldsymbol{\mu}_{s}^{\omega}\right\} .
\end{aligned}
$$

The set $\mathcal{A}_{j}\left(s^{\prime}\right)$ is the set of states in which student $j$ is assigned to $s^{\prime}$, while $\mathcal{B}_{j}(s)$ is the set of states in which $j$ has high enough priority to block with $s$. Let $\mathcal{C}_{j}\left(s^{\prime}, s\right)=\mathcal{A}_{j}\left(s^{\prime}\right) \cap \mathcal{B}_{j}(s)$. In words, $\mathcal{C}_{j}\left(s^{\prime}, s\right)$ is the set of states in which student $j$ is assigned to $s^{\prime}$, but could block with $s$.

Definition 2. Given a full matching $\boldsymbol{\mu}$, student-school pair $(j, s)$ is a blocking pair if there exists a state $\tilde{\omega}$ such that $\mathcal{C}_{j}\left(\boldsymbol{\mu}_{j}^{\tilde{\omega}}, s\right) \neq \varnothing$ and

$$
E\left[u_{j}^{\omega}(s) \mid \mathcal{C}_{j}\left(\boldsymbol{\mu}_{j}^{\tilde{\omega}}, s\right) \cap \mathcal{I}_{j}(\tilde{\omega})\right]>E\left[u_{j}^{\omega}\left(\boldsymbol{\mu}_{j}^{\tilde{\omega}}\right) \mid \mathcal{C}_{j}\left(\boldsymbol{\mu}_{j}^{\tilde{\omega}}, s\right) \cap \mathcal{I}_{j}(\tilde{\omega})\right] .
$$

Full matching $\boldsymbol{\mu}$ is stable if there are no blocking pairs. 
To understand this definition, fix a state $\tilde{\omega}$ and notice that the right side of the inequality is student $j$ 's expected utility from her current assignment, $\boldsymbol{\mu}_{j}^{\tilde{\omega}}$, given her information that the true state lies in the $\operatorname{set} \mathcal{C}_{j}\left(\boldsymbol{\mu}_{j}^{\tilde{\omega}}, s\right) \cap \mathcal{I}_{j}(\tilde{\omega})$. The left side of the inequality is $j$ 's expected utility from any other school $s$ with which she may potentially want to form a blocking pair. If the latter is greater than the former, then $(j, s)$ is a blocking pair and full matching $\boldsymbol{\mu}$ is not stable. Note also that for informed students, Definition 2 is equivalent to the definition of a classical blocking pair.

REMARK 1. Once incomplete information is introduced to matching, stability becomes harder to define because there is inherently some flexibility in the assumptions about the information that agents are able to condition on and certain modeling choices must be made. In particular, choices must be made in the following regard.

- What students know in equilibrium. In an equilibrium, each student knows the full matching induced by the equilibrium strategies. This allows students to condition on the states consistent with the information they have received and on whether the block will be successful. There is actually no flexibility here in modeling, as this is just a feature of equilibrium, but we want to emphasize it to avoid any confusion as to why students can condition on having higher priority than another student even when they do not directly observe this fact.

- What students directly observe about the realized matching. This is the dimension in which there is clear flexibility. We assume that students observes only their own assignment, but it is reasonable to examine other models. Liu (2019), for instance, considers a stability definition very closely related to ours, but allows agents to observe the entire matching.

- What additional information students could gather. There is flexibility here too. We assume that a student gathers no additional information while in the process of blocking. Essentially, this is an assumption that blocking is a static process in which a student proposes a block, it is accepted or rejected, and then the process ends. More generally, it is possible to model a dynamic process where the student learns additional information by proposing blocks that can then be used to propose additional blocks.

In summary, our definition of stability assumes that students explicitly observe only their own match, but take an equilibrium approach in that they have knowledge of the equilibrium mapping from states to matchings. The blocking definition takes a "oneshot deviation" approach that assumes the only explicit piece of information a student uses is his own assigned school combined with his knowledge of the equilibrium mapping from states to matchings. This of course is not the only way one might define stability in the presence of incomplete information. For instance, one could allow students to observe or gather more information after the initial matching is determined. This makes stability only harder to achieve and so, given the sparsity of analysis of incomplete information in the matching literature thus far, we think the assumptions we use are a natural way to proceed. 
The following example is instructive for understanding the concepts of full matchings and stability.

ExAmple 1. Suppose there are three students, $J=\left\{j_{1}, j_{2}, j_{3}\right\}$, with $j_{1}$ uninformed, and three schools, $S=\{A, B, C\}$, each with capacity 1 . The priorities at $A$ are $j_{3} \succ_{A} j_{1} \succ_{A} j_{2}$. There are four states $\Omega=\left\{\omega_{1}, \omega_{2}, \omega_{3}, \omega_{4}\right\}$ that are all equally likely. Consider the full matching $\boldsymbol{\mu}^{\omega}$ given in the table

\begin{tabular}{cccc}
\hline & $\boldsymbol{\mu}_{j_{1}}^{\omega}$ & $\boldsymbol{\mu}_{j_{2}}^{\omega}$ & $\boldsymbol{\mu}_{j_{3}}^{\omega}$ \\
\hline$\omega_{1}$ & $C$ & $A$ & $B$ \\
$\omega_{2}$ & $C$ & $A$ & $B$ \\
$\omega_{3}$ & $C$ & $B$ & $A$ \\
$\omega_{4}$ & $A$ & $B$ & $C$ \\
\hline
\end{tabular}

To understand when blocking pairs form, suppose that $j_{1}$ is matched to $C$ and is considering proposing blocking with $A$. As $j_{1}$ observes that she is matched to $C$, she rules out $\omega_{4}$ as a possible state $\left(\mathcal{A}_{j_{1}}(C)=\left\{\omega_{1}, \omega_{2}, \omega_{3}\right\}\right)$. As $j_{1}$ has lower priority than $j_{3}$ at $A$ but higher priority than $j_{2}, j_{1}$ knows that the blocking pair will not form in $\omega_{3}$, but rather only in states $\omega_{1}$ and $\omega_{2}\left(\mathcal{B}_{j_{1}}(A)=\left\{\omega_{1}, \omega_{2}\right\}\right)$. This means that $j_{1}$ will condition on the state being only either $\omega_{1}$ or $\omega_{2}\left(\mathcal{C}_{j_{1}}(C, A)=\left\{\omega_{1}, \omega_{2}\right\}\right)$. As the states are equally likely ex ante, $j_{1}$ 's updated belief is that each state has occurred with probability $1 / 2$, so $j_{1}$ will propose the block if and only if $\frac{1}{2} u_{j_{1}}^{\omega_{1}}(A)+\frac{1}{2} u_{j_{1}}^{\omega_{2}}(A)>\frac{1}{2} u_{j_{1}}^{\omega_{1}}(C)+\frac{1}{2} u_{j_{1}}^{\omega_{2}}(C)$.

It should be clear that given a mechanism $\psi$, any strategy profile $\sigma$ induces a full matching $\boldsymbol{\mu}(\sigma)$ defined by $\boldsymbol{\mu}^{\omega}(\sigma)=\psi(\sigma(\omega))$. This leads naturally to the next definition.

Definition 3. Mechanism $\psi$ is stable if there exists an equilibrium $\sigma$ such that the induced full matching $\boldsymbol{\mu}(\sigma)$ is stable.

\section{The Curse of ACCEPTANCE}

In this section, we highlight some of the problematic issues that arise in our model with incomplete information and uninformed students, and how they can be attributed to a so-called curse of acceptance. To fix ideas, we do this first in the context of one of the most familiar mechanisms, the student-proposing deferred acceptance (DA) mechanism. DA works as follows (for a more formal description, see, e.g., Abdulkadiroğlu and Sönmez (2003)).

Deferred acceptance Each student $j$ submits a strict preference ranking $P_{j}$. Begin with all students and schools unmatched.

Step $k$. Each unmatched student applies to her most preferred school that has not yet rejected her. Each school $s$ tentatively admits the $q_{s}$-highest priority students from the new applicants and previous tentatively admitted students. All students not tentatively admitted to a school are rejected. If some student is rejected, move to step $k+1$; otherwise, make all tentative assignments final and end the algorithm. 
DA has a rich history in the literature, going back to the foundational work of Gale and Lloyd (1962), and in their seminal paper, Abdulkadiroğlu and Sönmez (2003) propose DA as a promising solution for school choice. DA has since become one of the most widely used mechanisms in practice, including in such large school districts as New York City, Boston, and New Orleans, as well as many others in the United States and around the world. DA has been explored in depth in the literature and is well known to satisfy many desirable properties in the standard model: most importantly, it is (i) strategy-proof, (ii) classically stable, and (iii) Pareto dominates any other classically stable mechanism.

Since DA is so popular, and most of the prior work and positive results for DA work in a private values setting, it becomes particularly important to understand what happens when these assumptions are relaxed. We start in this section by looking at the following special case of our model, which we call the common ordinal preferences model: The state space is $\Omega=\mathcal{P}$ and the probability distribution $\operatorname{Pr}$ over $\Omega$ is the uniform distribution. The state should be interpreted as the common ordinal preference held by every student, and the uniform distribution ensures that the uninformed students are truly uninformed ex ante, which will be important for our positive results below. Finally, every student $j$ shares a common utility function $u_{j}^{\omega}(s)=M-\operatorname{rank}_{\omega}(s)$. This functional form is for concreteness only. Our positive results are stated in terms of first-order stochastic dominance, and so any utility function that induces the common ordinal preferencepossibly differing across students-obtains the same results.

School choice problems in this model are specified by a set of students (who are uninformed), a set of schools, and the school capacities/priorities. Example 2 shows that when there is even one student who is not informed about her preferences, DA is no longer stable.

Example 2. There are four students: $I=\left\{j_{1}, j_{2}, j_{3}\right\}$ are informed and $U=\left\{j_{4}\right\}$ is uninformed. There are also four schools, $S=\{A, B, C, D\}$, each with capacity 1 . The priority structure is

\begin{tabular}{cccc}
\hline$\succ_{A}$ & $\succ_{B}$ & $\succ_{C}$ & $\succ_{D}$ \\
\hline$j_{1}$ & $j_{1}$ & $j_{3}$ & $j_{2}$ \\
$j_{2}$ & $j_{3}$ & $j_{1}$ & $j_{1}$ \\
$j_{3}$ & $j_{2}$ & $j_{4}$ & $j_{4}$ \\
$j_{4}$ & $j_{4}$ & $j_{2}$ & $j_{3}$ \\
\hline
\end{tabular}

After receiving their signals, each student submits a preference list and the final matching is computed using the DA algorithm. It has been shown (McVitie and Wilson 1971, Dubins and Freedman 1981) that an equivalent way to run DA is to arbitrarily choose one unmatched student and have him apply to his most preferred school where he have not yet been rejected. The order in which students are chosen to apply does not matter; in particular, we consider running DA starting with $j_{1}, j_{2}$, and $j_{3}$, and then, when they are all tentatively matched, have $j_{4}$ enter the market.

For the informed students, the truthful strategy $\sigma_{j}(\omega)=\omega$ is weakly dominant, so that, e.g., in state $\omega=D A B C$, students $j_{1}, j_{2}$, and $j_{3}$ will all report $\sigma_{j}(\omega)=D A B C$. Now 
note that before $j_{4}$ makes her first application in this method of DA, $j_{1}, j_{2}$, and $j_{3}$ are tentatively matched to the best three schools in every state. Given the priority structure, student $j_{4}$ will just get matched to the worst school unless $j_{2}$ is tentatively matched to $C$ or $j_{3}$ is tentatively matched to $D$. Of the 24 states, this happens in only two of them: state $\omega^{\prime}=A B C D$, where $j_{2}$ is matched to $C$, and state $\omega^{\prime \prime}=B A D C$, where $j_{3}$ is matched to $D$. The final matching in these two states will depend on what preference relation $j_{4}$ reports. If $j_{4}$ reports a preference relation $P_{j_{4}}$ with $C P_{j_{4}} D$, then $j_{4}$ will match to $C$ in both $\omega^{\prime}$ and $\omega^{\prime \prime}$, whereas if $j_{4}$ reports a preference relation $P_{j_{4}}^{\prime}$ with $D P_{j_{4}}^{\prime} C$, then $j_{4}$ will match to $D$ in both $\omega^{\prime}$ and $\omega^{\prime \prime}$. The key point to notice is that no matter what she submits, $j_{4}$ will receive the same assignment in $\omega^{\prime}$ and $\omega^{\prime \prime}$. In one of these states, this assignment will be the third-best school, and in the other, it will be the worst. Combining this with the previous discussion, we see that $j_{4}$ will get the worst school in 23 states and the third-best school in one state, regardless of what she reports.

We claim that for either report $\left(P_{j_{4}}\right.$ or $P_{j_{4}}^{\prime}$ ), the resulting full matching is not stable. Suppose $j_{4}$ reports $C P_{j_{4}} D$ and observes that she is matched to $C$. In equilibrium, $j_{4}$ is matched to $C$ in seven states: the six states where $C$ is worst and $\omega^{\prime}=A B C D$. But $j_{3}$ is only matched to $D$ in $\omega^{\prime \prime}=B A D C$, so if $j_{4}$ proposes a block with $D$, it will be accepted only in state $\omega^{\prime \prime}$. Using the notation introduced above, we have

$$
\begin{aligned}
\mathcal{A}_{j_{4}}(C) & =\{A B C D, A B D C, A D B C, B A D C, B D A C, D A B C, D B A C\}, \\
\mathcal{B}_{j_{4}}(D) & =\{B A D C\}, \\
\mathcal{C}_{j_{4}}(C, D) & =\{B A D C\} .
\end{aligned}
$$

For any state $\tilde{\omega}$, where $j_{4}$ is matched to $C$ in equilibrium, we thus have

$$
1=E\left[u_{j_{4}}^{\omega}(D) \mid \mathcal{C}_{j_{4}}\left(\boldsymbol{\mu}_{j_{4}}^{\tilde{\omega}}, D\right) \cap \mathcal{I}_{j_{4}}(\tilde{\omega})\right]>E\left[u_{j_{4}}^{\omega}\left(\boldsymbol{\mu}_{j_{4}}^{\tilde{\omega}}\right) \mid \mathcal{C}_{j_{4}}\left(\boldsymbol{\mu}_{j_{4}}^{\tilde{\omega}}, D\right) \cap \mathcal{I}_{j_{4}}(\tilde{\omega})\right]=0,
$$

where $\boldsymbol{\mu}$ is the full matching induced by DA with the specified equilibrium strategies. So $j_{4}$ benefits from the block and so $\mu$ is not stable. If $j_{4}$ were instead to report $P_{j_{4}}^{\prime}$ with $D P_{j_{4}}^{\prime} C$, a symmetric argument shows that she will want to propose blocking with $C$ when she matches to $D$. In summary, we have shown the following situation.

Proposition 1. There exist preferences and a priority structure such that no equilibrium of the deferred acceptance mechanism is stable.

The above impossibility result is in the spirit of most such results in the school choice and matching literature, which construct specific counterexamples to show certain properties do not hold in general. There are two ways to proceed from this: one is to look for other stable mechanisms besides DA, while the other is to look for conditions under which DA is stable. Starting in the next section, we discuss both approaches; however, before getting there, we note that there are even larger problems with DA besides instability. In the last example, the uninformed student gets the worst possible school in 23 of the 24 states. Of course, part of the reason for this is that she has relatively low priority at each school. But, as we see later, no matter her priority, she always does worse than average. 
These two features-no stability and generally poor outcomes for the uninformed students-can be attributed to a so-called curse of acceptance: uninformed students do not know which schools are the good schools, but upon seeing their assignment, they update their beliefs about the quality of the school they were assigned downward. The reason is that they know that the more informed students know which schools are the good schools and end up taking them for themselves. This leaves the less informed students with the low quality schools, which not only makes them worse off from a welfare perspective, but also leads to instability.

This still is not the end of the story, as we have yet to consider the incentives for preference reporting to the mechanism. Though DA is strategy-proof in the standard model, this is no longer feasible in our model, and it turns out that without it, determining the equilibrium can be quite complicated. In the previous example, every strategy for $j_{4}$ yielded the same expected utility and, therefore, every strategy was an equilibrium. This is just an artifact of the example, however; usually determining an optimal strategy is far more complicated and depends very much on the details of the full priority structure. This can make playing the mechanism very difficult for some parents, which is unappealing to many school districts (see Pathak and Sönmez (2008)).

The next example clearly illustrates this point. It considers two different problems, which barely differ in their primitives but have vastly different equilibria. Furthermore, the example shows that a student's own priority is not sufficient for determining her equilibrium strategy (i.e., reporting a preference list that orders schools by one's priorities at them is generally not an equilibrium strategy for the uninformed students).

ExAmple 3. The students and schools are the same as in Example 2. We consider two more examples with slightly different priority structures:

\begin{tabular}{ccccccccc}
\hline$\succ_{A}$ & $\succ_{B}$ & $\succ_{C}$ & $\succ_{D}$ & & $\succ_{A}^{\prime}$ & $\succ_{B}^{\prime}$ & $\succ_{C}^{\prime}$ & $\succ_{D}^{\prime}$ \\
\hline$j_{3}$ & $j_{2}$ & $j_{2}$ & $j_{2}$ & & $j_{3}$ & $j_{2}$ & $j_{2}$ & $j_{2}$ \\
$j_{4}$ & $j_{1}$ & $j_{1}$ & $j_{1}$ & & $j_{4}$ & $j_{1}$ & $j_{1}$ & $j_{3}$ \\
$j_{2}$ & $j_{4}$ & $j_{3}$ & $j_{3}$ & & $j_{2}$ & $j_{4}$ & $j_{3}$ & $j_{1}$ \\
$j_{1}$ & $j_{3}$ & $j_{4}$ & $j_{4}$ & & $j_{1}$ & $j_{3}$ & $j_{4}$ & $j_{4}$ \\
\hline
\end{tabular}

Priority structure $\succ^{\prime}$ alters $\succ$ only by switching the priority of $j_{1}$ and $j_{3}$ at school $D$ (in particular, $j_{4}$ 's priorities have not changed). It can be checked (following the same analysis as in Example 2, so we omit the calculations) that under $\succ$, the uninformed student $j_{4}$ 's equilibrium strategies consist of any report that ranks $B P_{j_{4}} A$ (the rankings of $C$ and $D$ are irrelevant). However, under $\succ^{\prime}$, the equilibrium strategies for $j_{4}$ consist of any report where $A P_{j_{4}} B$. So just switching the priorities of two students at a schoolwho both have higher priority than $j_{4}$ and to which $j_{4}$ is never matched unless it is the worst school-completely reverses the equilibrium strategies. In one case, $j_{4}$ should favor the school at which they have second-highest priority, but in the other case, they should favor the school at which they have third-highest priority. There is no way for $j_{4}$ to determine her equilibrium strategy without considering the full priority structure of all students. 


\section{Mechanism DESIgN VerSUS PRIORITY DESIgN}

Proposition 1 shows that DA is not stable in general. There are two ways to proceed from this. The first is to ask whether there is some other mechanism that is always stable for any priority structure (including the counterexample in the last section); the second is to ask whether there are certain classes of priority structures for which we recover stability of DA. We look at both of these questions in turn. First, we show that there is a stable mechanism by construction, but also argue that any stable mechanism inherently suffers from other flaws that prevent it from being a practically useful solution. Motivated by this impossibility result, we turn to the latter approach of looking at the problem from a priority design perspective.

First, we show the existence of a mechanism that is formally stable in the common ordinal preference model of the previous section by introducing the following statelearning mechanism.

State-learning mechanism. Fix an arbitrary preference profile $\bar{P}$ and matching $\bar{\mu}$. If there exists some $\tilde{P} \in \mathcal{P}$ such that $P_{j} \notin\{\bar{P}, \tilde{P}\}$ for at most one $j \in J$ (the case $\tilde{P}=\bar{P}$ is allowed, in which case this set is a singleton), then output a matching that is classically stable with respect to $\tilde{P}$; otherwise, output the matching $\bar{\mu}$.

The idea behind the mechanism is that informed students (who know $\omega$ ) submit the preference $P_{j}=\omega$, while uninformed students submit $P_{j}=\bar{P}$. These reports are an equilibrium because any unilateral deviation is ignored. As long as $|I| \geq 3$, this allows the mechanism to learn the state and, therefore, also learn the common preference ranking of all agents. Once the mechanism knows the common preference, it can choose a classically stable matching for that preference ranking, which will imply that the corresponding full matching $\boldsymbol{\mu}$ is stable.

While the state-learning mechanism is formally stable, we do not believe it is a useful mechanism for practical market design. Intuitively, the reason is that such mechanisms override the stated preferences of some students, replacing them with different preferences. To make this concrete, consider again Example 2, in which there is a unique stable full matching $\mu$. Recall that the key states in this example are state $\omega^{\prime}=A B C D$, where $\boldsymbol{\mu}_{j_{4}}^{\omega^{\prime}}=C$ and $\boldsymbol{\mu}_{j_{2}}^{\omega^{\prime}}=D$, and state $\omega^{\prime \prime}=B A D C$, where $\boldsymbol{\mu}_{j_{4}}^{\omega^{\prime \prime}}=D$ and $\boldsymbol{\mu}_{j_{3}}^{\omega^{\prime \prime}}=C$. In all states $\omega \neq \omega^{\prime}, \omega^{\prime \prime}$, the matching is uniquely determined by stability constraints for the informed students, as the informed students must be matched to the best three schools. For states $\omega^{\prime}$ and $\omega^{\prime \prime}$, the only other possibilities for students are for $j_{2}$ to match to $C$ in $\omega^{\prime}$ and/or $j_{3}$ to match to $D$ in $\omega^{\prime \prime}$. But, as shown earlier, the resulting full matchings are not stable and so $\boldsymbol{\mu}$ is the unique stable full matching. Suppose that, for instance, the state is $\omega^{\prime}$, and $j_{1}, j_{2}$, and $j_{3}$ report $P_{j}=A B C D$ while $j_{4}$ reports some preference $P_{j_{4}}$ where $D P_{j_{4}} C$. Since there is a unique stable full matching, any stable mechanism must assign $j_{4}$ to $C$ and $j_{2}$ to $D$. On the one hand, there is nothing wrong with this assignment in the model of common ordinal preferences, because the mechanism knows that the report of $j_{4}$ does not reflect $j_{4}$ 's true preference. On the other hand, this requires a strong assumption that the designer is certain in his knowledge about the correlation structure in the agents' preferences; that is, while he may not know the state, the designer at 
least knows the mapping from states to agent utilities. ${ }^{3}$ If the designer is wrong about his assessment of the agent's utility functions and actually $j_{4}$ 's report does reflect $j_{4}$ 's true preference, then the matching implemented is undesirable: $j_{2}$ and $j_{4}$ would like to trade schools and so the matching is not efficient. Even further, this trade would not violate stability, as neither $j_{1}$ nor $j_{3}$ would want to block after the trade was implemented, and so the final matching is not even constrained efficient. Constrained efficiency here means Pareto efficient within the set of stable matchings and is one of the celebrated properties that is satisfied by the DA mechanism.

While a mechanism designer may (quite justifiably) expect that student preferences are highly correlated and some students are more informed than others, they are unlikely to know the correlation structure exactly or precisely which students are informed or uninformed. This suggests that a "good" mechanism should take the reported preferences into account, rather than simply disregarding them, as the state-learning mechanism (and indeed, any stable mechanism) does. Unfortunately, as our example shows, there is a no way to ensure stability without risking ending in an undesirable final allocation if the mechanism has wrongly identified the information and preferences of students; in other words, stable mechanisms are not robust to misspecification.

To make this point more rigorously, we say that, given some property $\alpha$, a mechanism $\psi$ satisfies $\alpha$ robustly if it satisfies $\alpha$ in the full information model in which each student is informed $(J=I)$. The main property that we are concerned with is constrained efficiency. Formally, given a matching $\mu$ and a profile of ordinal preferences $P$, $\mu$ is said to be constrained efficient with respect to $\boldsymbol{P}$ if for any two students $j$ and $j^{\prime}$ such that $\mu_{j^{\prime}} P_{j} \mu_{j}$ and $\mu_{j} P_{j^{\prime}} \mu_{j^{\prime}}$, there exists $j^{\prime \prime} \neq j, j^{\prime}$ such that either $\mu_{j^{\prime}} P_{j^{\prime \prime}} \mu_{j^{\prime \prime}}$ and $j^{\prime \prime} \succ_{\mu_{j^{\prime}}} j$ or $\mu_{j} P_{j^{\prime \prime}} \mu_{j^{\prime \prime}}$ and $j^{\prime \prime} \succ_{\mu_{j}} j^{\prime}$. A mechanism $\psi$ is constrained efficient if there exists an equilibrium $\sigma$ such that the induced matching $\boldsymbol{\mu}^{\omega}(\sigma)$ is constrained efficient with respect to $\sigma(\omega)$ for each $\omega$; if no such equilibrium exists, then $\psi$ is said to be constrained inefficient. In the standard model, constrained efficiency is equivalent to student optimality subject to stability, and one of the celebrated properties of deferred acceptance is that it produces the so-called student-optimal stable match.

For the next impossibility result, we go beyond DA (and beyond strategy-proof mechanisms) to consider a natural class of mechanisms that we call truthful mechanisms, in which there is an equilibrium in which the informed students all play the truthful strategy, $\sigma_{j}^{t}(\omega)=P_{j}(\omega)$. This is not too restrictive, due to a version of the revelation principle: If $\psi$ is any mechanism and $\sigma^{*}$ is an equilibrium of the game induced by $\psi$, then there exists a mechanism $\psi^{\prime}$ where $\left(\left(\sigma_{j}^{t}\right)_{j \in I},\left(\sigma_{j}^{*}\right)_{j \in U}\right)$ is an equilibrium of the game induced by $\psi^{\prime}$ and the resulting matchings are the same in every state. ${ }^{4}$ The truthful equivalent of any mechanism seems desirable, since informed students must just

\footnotetext{
${ }^{3}$ Similar issues arise with "state-verification mechanisms" in many other mechanism design contexts. While this appears less in the matching literature, which has so far relatively little work on interdependent values, one exception is Bikhchandani (2017), who provides an incentive compatible and stable mechanism that works in a similar way. Like us, he seems skeptical of its usefulness, writing that "it is required that the mechanism designer knows agents' utility functions, which is a strong assumption."

${ }^{4}$ The revelation principle is standard, but we provide a proof in the Appendix that gives the (straightforward) arguments needed to accommodate both informed and uninformed students.
} 
report their true preferences, rather than coordinate on some complicated preference reporting equilibrium.

Proposition 2. There exist preferences and a priority structure such that any stable and truthful mechanism is robustly constrained inefficient.

Because of this impossibility, in the remainder of the paper, we take the second approach and analyze the problem from a priority design perspective. We show how this can be used to recover positive results in the widely used DA mechanism. In particular, we provide a class of priority structures under which DA is stable, and the equilibrium strategies are simple and focal. Further, DA is robustly constrained efficient in the sense just discussed.

Looking at conditions on priorities under which desirable properties hold is a common approach in the theoretical literature on priority-based object allocation. Ergin (2002) asks which priority structures ensure that DA is Pareto efficient (rather than just constrained efficient), an analysis which is extended by Kojima (2013) to multi-unit demand. Ehlers and Erdil (2010), Ehlers and Westkamp (2018), and Han (2018) study related questions of priority design when weak priorities are allowed. Doval (2015) looks at dynamic matching markets and proposes a theory of dynamic stability. Since dynamically stable matchings need not exist in general priority-based allocation problems, she asks which priority structures guarantee the existence of a dynamically stable matching. Ashlagi and Gonczarowski (2018) provide conditions under which DA will be obviously strategy-proof in the sense of $\mathrm{Li}(2017) .^{5}$

While deepening our theoretical understanding of matching mechanisms, from a practical market design perspective, questions of priority design are relevant only if the designer has some control over them in practice. Indeed, school districts often do have some control over the design of their priority structures and some have attempted to (re)design their priorities to achieve particular objectives. For example, Dur et al. (2018) and Pathak and Shi (2017) explore consequences of different design decisions relating to the development of walk zones in Boston, a city which has taken an active role in the design of its priorities. Roughly, schools are divided into quality tiers, and a student's menu consists of the two closest tier 1 schools, the four closest tier 2 schools, etc. If a school is not on a student's menu, then she is not allowed to list it, which is effectively a way to give higher priority at the school to those who are allowed to apply to it. Note that all of the aforementioned papers still work in the standard framework of perfectly informed students. In the remained of this paper, we analyze the consequences when perfect information is relaxed.

\section{SeCure SCHOols}

Following the discussion in the previous section, we now introduce a class of priority structures for which DA has good properties: it is stable, the equilibrium strategies is

\footnotetext{
${ }^{5}$ Kesten (2006) and Troyan (2019) analyze complementary questions of priority design for top trading cycles, while Kumano (2013) does so for the Boston mechanism.
} 
simple, and the uninformed students is protected from the curse of acceptance, which improves welfare. We say that school $s$ is a secure school for student $j$ if $\mid\left\{j^{\prime} \in J: j^{\prime} \succsim s\right.$ $j\} \mid \leq q_{s}$. In words, a secure school is one with enough seats for $j$ and every student who has higher priority than $j$. It is useful to point out that while secure schools may seem reminiscent of "neighborhood schools," secure schools need not be geographically determined. While geography does happen to be a commonly used criterion in practice, it is not the only possibility; indeed, some places have begun to recognize it may be better to use other criteria besides geography (see the discussion of Boston in the previous section). We abstract away from such considerations to focus more squarely on the asymmetric information problem, and in the rest of this section, we show how secure schools allow uninformed students to guard themselves against the curse of acceptance. ${ }^{6}$

How do secure schools help the uninformed? The problem for them is the curse of acceptance, and a secure school allows for them to have a default option that they can get in every state. Hence, the curse is entirely eliminated by allowing them to expect to get average utility, rather than always being left with the worst schools in every state. Theorem 1 formalizes this intuition to all markets in the common ordinal preferences model. This assumption is necessary to get a clear result in the discrete model, as the proofs rely on combinatoric arguments that quickly become intractable without it. However, the intuition applies more broadly, and in the next section, we use a continuum model to analyze the more general case of heterogeneous (but correlated) preferences.

For the priority structure, we assume that each student has at least one secure school, but otherwise, the priority structure is arbitrary. Define the strategy profile $\sigma_{j}^{*}(\omega)$ as follows: for all informed $j \in I, \sigma_{j}^{*}(\omega)=\omega$, and for all uninformed $j \in U$, $\sigma_{j}^{*}(\omega)=\tilde{P}_{j}$ for all $\omega$, where $\tilde{P}_{j}$ is any preference ranking that lists one of $j$ 's secure schools first. As discussed above, following $\sigma_{j}^{*}$ is weakly dominant for the informed students; i.e., after observing their signal, they just report their true preferences. Our theorem shows that taking their secure school is also optimal for the uninformed students in a first-order stochastic dominance sense. (A strategy will induce a distribution over the number of states where $j$ gets the best school in that state, the second-best school, the third-best school, etc. This is the distribution to which the first-order stochastic dominance result in the theorem applies.)

THEOREM 1. Consider any market in the common ordinal preferences model and assume that every student has a secure school. Then j's outcome from the deferred acceptance mechanism under profile $\left(\sigma_{j}^{*}, \sigma_{-j}^{*}\right)$ first-order stochastically dominates her outcome un$\operatorname{der}\left(\sigma_{j}^{\prime}, \sigma_{-j}^{*}\right)$ for any other strategy $\sigma_{j}^{\prime}$ that $j$ could choose. Thus, $\sigma^{*}$ is an equilibrium strategy profile of deferred acceptance.

\footnotetext{
${ }^{6}$ Antler (2015) studies a different type of preference interdependency in one-to-one two-sided matching between men and women, and also shows failure of stability of standard DA. While the model is quite different (preferences depend endogenously on how agents are ranked by those on the other side), his solution to restore stability is a modified DA mechanism that bears a striking resemblance to secure schools. In his modified DA mechanism, each woman is allowed to rank only one man. Thus, in equilibrium, men are able to guarantee being matched to certain women as long as they rank them first (it is a complete information game, so the men know the women's strategies in equilibrium), just as with students and secure schools in our model.
} 
While Theorem 1 constructs one equilibrium, it is a natural equilibrium. When uninformed students do not have a secure school, determining equilibrium strategies for the uninformed students can be quite complicated, as seen in Example 3. Designing priorities such that every student has a secure school results in equilibrium strategies that are focal and very simple to compute: informed students follow the familiar truthtelling strategy, while uninformed students simply take their secure school. Doing so is optimal because it protects them from the curse of acceptance identified previously.

Furthermore, we think that secure schools may be helpful in this environment not only because there exists an equilibrium in simple strategies, but also because the full matching induced by these strategies is stable. This is stated in the following theorem.

THEOREM 2. Consider any market in the common ordinal preferences model and suppose every student has a secure school. The equilibrium strategy $\sigma^{*}$ induces a stable matching and, therefore, the deferred acceptance mechanism is stable.

Each uninformed student receives his secure school, so he learns nothing from observing his own match. It is tempting to think that this immediately implies that the full matching is stable, but there is more to the argument than that. The students do not learn anything explicitly from the realized matching, but because they know the equilibrium mapping from states to matchings and the priorities, when calculating their expected utility from proposing a block with a school $s$, they can still condition on the set of states in which the block is accepted (recall the set $\mathcal{B}_{j}$ above). If, in equilibrium, the block is accepted in states where the student is better off but not in states where they are worse off, the matching may not be stable. Thus, to prove the theorem, it must be shown that this cannot happen. Intuitively, the reason is that the lowest priority student initially assigned to $s$ has lower priority according to $s$ 's priority ranking in states where $s$ is worse (according to the common ordinal preference). Thus, if student $j$ 's proposed block is accepted in some state where $s$ is the $k$ th best school, then it will also be accepted when $s$ is the $(k+1)$ th best school, the $(k+2)$ th best school, etc., down to the worst school. The complete details of the argument can be found in the Appendix.

\section{Heterogeneous Preferences}

While preferences over schools are likely correlated in the real world, they are almost certainly not perfectly correlated, which raises the question of how our results extend to this setting. The proof strategies used for the discrete economies in the previous section rely on combinatoric arguments that explode and quickly become intractable when preferences are correlated but heterogeneous. However, the intuition for the curse of acceptance still holds when preferences are partially correlated. We can obtain formal results by moving to continuum economies, which have recently been receiving significant interest in the matching literature because they simplify analysis considerably while still providing useful insights (see, for example, Miralles (2009), Abdulkadiroğlu et al. (2015), Azevedo and Leshno (2016)).

Continuum economy with correlated preferences model. There is still a finite set of schools $S=\left\{s_{1}, \ldots, s_{M}\right\}$, but each school now has a unit mass of seats to fill. There is a 
total mass $M$ of students. As in the previous section, the underlying state space is $\Omega=\mathcal{P}$ and $\mathrm{Pr}$ is the uniform distribution (which, recall, is necessary to ensure that uninformed students are truly uninformed ex ante). However, the state now no longer determines a common ordinal preference for all agents; rather, the state is interpreted as a ranking of the intrinsic qualities of the schools, and students may have individual rankings that differ from this due to idiosyncracies. ${ }^{7}$ Formally, we model each state $\omega$ as determining a measure $\lambda_{\omega}$ over $\mathcal{P}$, where, for any ordinal ranking $P, \lambda_{\omega}(P)>0$ is the measure of students who have ordinal preferences $P$ in state $\omega$ with $\sum_{P \in \mathcal{P}} \lambda_{\omega}(P)=M$. In this section, we write states as $\omega=\left(s_{\pi(1)}, s_{\pi(2)}, \ldots, s_{\pi(M)}\right)$, where $\pi$ is the permutation of the set $\{1, \ldots, M\}$ such that $\operatorname{rank}_{\omega}\left(s_{\pi(k)}\right)=k$ for each $k=1 \ldots, M$. Student $j$ 's cardinal preference for school $s$, given that she has ordinal preferences $P_{j}$, is $u_{j}^{P_{j}}(s)=v\left(\operatorname{rank}_{P_{j}}(s)\right)$, where $v$ is some strictly decreasing function.

Say that a preference ranking $P$ is closer to state $\omega$ than $P^{\prime}$ if there exist two schools $s$ and $s^{\prime}$ such that $\operatorname{rank}_{\omega}(s)<\operatorname{rank}_{\omega}\left(s^{\prime}\right)$ and $\operatorname{rank}_{P}(s)<\operatorname{rank}_{P^{\prime}}(s)$, and for all other $s^{\prime \prime} \neq s, s^{\prime}$, $\operatorname{rank}_{P}\left(s^{\prime \prime}\right)=\operatorname{rank}_{P^{\prime}}\left(s^{\prime \prime}\right)$; in other words, $P$ and $P^{\prime}$ differ only in where they rank $s$ and $s^{\prime}$, and $P$ ranks the two in the same way as $\omega$. We make the following assumptions on $\lambda_{\omega}$.

(i) We have $\lambda_{\omega}(P) \neq \lambda_{\omega}\left(P^{\prime}\right)$ for all $P \neq P^{\prime}$.

(ii) Let $\pi, \tilde{\pi}$, and $\hat{\pi}$ be any three permutations of the set $\{1, \ldots, M\}$. For the two states $\omega=\left(s_{\pi(1)}, \ldots, s_{\pi(M)}\right)$ and $\tilde{\omega}=\left(s_{\tilde{\pi}(1)}, \ldots, s_{\tilde{\pi}(M)}\right)$, and two preferences $P=$ $\left(s_{\hat{\pi}(1)}, s_{\hat{\pi}(2)}, \ldots, s_{\hat{\pi}(M)}\right)$ and $\tilde{P}=\left(s_{\tilde{\pi}\left(\pi^{-1}(\hat{\pi}(1))\right)}, s_{\tilde{\pi}\left(\pi^{-1}(\hat{\pi}(2))\right)}, \ldots, s_{\tilde{\pi}\left(\pi^{-1}(\hat{\pi}(M))\right)}\right)$, we have $\lambda_{\omega}(P)=\lambda_{\tilde{\omega}}(\tilde{P})$.

(iii) If $P$ is closer to state $\omega$ than $P^{\prime}$, then $\lambda_{\omega}(P)>\lambda_{\omega}\left(P^{\prime}\right)$.

The first condition rules out knife-edge cases where two preference profiles have exactly the same measure of students, which is not a generic property. The second says that the distribution of preferences depends only on the rankings of schools relative to their states, and not on their labels. For example, if the schools are $A, B$, and $C$, then the measure of students with preferences $P: B, C, A$ in state $\omega=(A, B, C)$ equals the measure of students with preferences $\tilde{P}: C, A, B$ in state $\tilde{\omega}=(B, C, A)$. The last condition, our assumption on how preferences are correlated, captures the idea that higher quality schools (as determined by the state) should be more popular in aggregate: if $P$ is closer to state $\omega$ than $P^{\prime}$, then there are more students with preference $P$ than preference $P^{\prime} .8$

For tractability, we move to the case of "weak priorities," where lotteries are used to determine final priority and immediately introduce secure schools. We assume each

\footnotetext{
${ }^{7}$ In this section, we slightly abuse notation and, when not referring to a specific student $j$, use $P$ to denote a generic preference ranking over the schools.

${ }^{8}$ One concrete way to model preferences in a finite market that satisfies these assumptions is to assume an underlying probability distribution Pr such that in each state $\omega=\left(s_{\pi(1)}, \ldots, s_{\pi(M)}\right)$ we have that $\tilde{\operatorname{Pr}}\left(s_{\pi(1)}\right)>\tilde{\operatorname{Pr}}\left(s_{\pi(2)}\right)>\cdots>\tilde{\operatorname{Pr}}\left(s_{\pi(M)}\right)$, and then we have each student $j$ draw her preferences $P_{j}$ via repeated draws from Pr, removing repetitions, until all $M$ schools have been drawn. This procedure induces a probability distribution over all preferences $\mathcal{P}$, and the measure $\lambda$ is the analogue of this probability distribution in the continuum model. This procedure for drawing preferences is commonly used in the literature (see, e.g., Immorlica and Mahdian (2005), Kojima and Pathak (2009), and Kojima et al. (2013)).
} 
student has exactly one secure school and for each school $s$, there is a measure 1 of students such that $s$ is their secure school. For the formal model, each student $j$ is endowed with priority numbers $\ell_{j}(s) \in[0,1]$, one for each school $s$, and $j$ 's priority score at $s$ is $\ell_{j}(s)+1$ for each school other than $j$ 's secure school and $\ell_{j}(s)$ for $j$ 's secure school (where we use the convention that smaller numbers are better priority). For any $x \in[0,1]$, the measure of students with $\ell_{j}(s) \leq x$ is equal to $x$ for each $s$. All students observe their own priority numbers before submitting their preferences. The uniform distribution is for convenience only (any distribution with a smooth density would suffice) and the equilibrium results below continue to hold whether students observe their priority numbers or not.

Of the total measure $M$ of students, a measure $\nu$ are informed, and the remaining $M-\nu$ are uninformed. A student's information type, preferences, and priorities are independent; in other words, in each state $\omega$, the measure of informed students who have preferences $P$ and priority numbers $\ell_{j}(s) \leq x$ at school $s$ is $\nu \lambda_{\omega}(P) x$ and the measure of respective uninformed students is $(M-\nu) \lambda_{\omega}(P) x$.

REMARK 2. Endowing students with preferences and priorities that add up to the measures given above is a way to get around a technical problem with perhaps the more natural way one would want to define the model in a finite world, which is to have each student $j$ independently draw an ordinal preference $P_{j}$ from some distribution over $\mathcal{P}$ (which may depend on the state; see footnote 8) and a priority score at each school. The technical problem is that when we move to the continuum, realizations of such random draws may result in the set of students with preference $P$ and priority scores less than $x$ being nonmeasurable. The model as formally defined does not suffer from this problem. Similar approaches are taken by Miralles (2009) and Abdulkadiroğlu et al. (2015) to deal with this issue.

As before, each student submits an ordinal preference relation, which then is turned into a matching using the DA mechanism. Intuitively, the deferred acceptance mechanism works in the same way as in the discrete case: students apply starting at the top of their (submitted) preference list, schools tentatively admit a measure equal to its capacity of students who have the highest priority, rejected students apply to their next most preferred school, etc. However, Abdulkadiroğlu et al. (2015) show that in a continuum model, the DA output can be characterized by a unique vector of cutoffs $\left(\bar{\ell}_{s_{1}}, \ldots, \bar{\ell}_{s_{M}}\right)$ (see also Azevedo and Leshno (2016)). Each student $j$ is then assigned to her most preferred school for which her priority score is lower than the school's cutoff. Any individual student has no ability to affect the cutoffs and so effectively acts as a "price-taker." This simplifies the strategic analysis, since to analyze the equilibrium outcomes of DA, we only need to understand the structure of these cutoffs. The next proposition provides the key property of the cutoffs on which the subsequent equilibrium results are built. As above, let $\sigma^{*}$ be a strategy profile where all informed students report their true preferences and uninformed students report their secure school first in their preference list. 
Proposition 3. Consider any market in the continuum economy with correlated preferences model. Fix a state $\omega=\left(s_{\pi(1)}, s_{\pi(2)}, \ldots, s_{\pi(M)}\right)$ and suppose the students play $\sigma^{*}$. Then the deferred acceptance cutoffs satisfy $1<\bar{\ell}_{s_{\pi(1)}}<\bar{\ell}_{s_{\pi(2)}}<\cdots<\bar{l}_{s_{\pi(M)}} \leq 2$.

This proposition gives a characterization of the equilibrium cutoffs under the proposed strategies. Intuitively, the cutoffs are smaller (and so the school is harder to get into) for the schools that are more likely to be popular. Indeed, if any of these inequalities were reversed, then there is some school that is both more popular in aggregate and is easier to get into. The proof in the Appendix reaches a contradiction by showing that this would result in some school being over capacity. Recall also that priority scores at any school $s$ range from 0 to 2 , with scores in $[0,1]$ reserved for students for whom $s$ is their secure school. Since there is only a unit mass of students for which this is true and all schools must be filled to capacity (because the total measure of students is equal to the total capacity of the schools), all cutoffs will be greater than 1 and the highest cutoff will be 2 .

We can now use Proposition 3 to find an equilibrium of the preference submission game. For the informed students, truthful reporting continues to be a weakly dominant strategy. What is left to show is that for an uninformed student, listing her secure school $\bar{s}$ first gives a higher expected utility than any other strategy. The proof of the following theorem is technical, but of all the many cases, the one that matters is intuitive. Consider any other $s^{\prime} \neq \bar{s}$, and two states $\omega$ and $\omega^{\prime}$ such that $\operatorname{rank}_{\omega}(\bar{s})<\operatorname{rank}_{\omega}\left(s^{\prime}\right)$ and $\operatorname{rank}_{\omega^{\prime}}\left(s^{\prime}\right)<$ $\operatorname{rank}_{\omega^{\prime}}(\bar{s})$ (and the ranks of the other schools are identical). If $j$ submits her secure school at the top of her list, she will get it in both states. If she instead puts $s^{\prime}$ above her secure school, then it is possible for her priority number to only be small enough to get it when $s^{\prime}$ is less popular (i.e., in state $\omega$ ), where, by Proposition 3 , the cutoff to get into $s^{\prime}$ is larger than in state $\omega^{\prime}$, giving her the worse school in both states (i.e., $s^{\prime}$ in state $\omega$ and $\bar{s}$ in state $\left.\omega^{\prime}\right)$

THEOREM 3. Consider any market in the continuum economy with correlated preferences model. The strategy profile $\sigma^{*}$ is an equilibrium of the deferred acceptance mechanism.

What about stability? As in the discrete case, there is no learning from one's observed match by uninformed students. However, we still need to address the issue of conditioning on a potential block being accepted. This turns out to be much simpler in this setting than in the discrete case, because, by Proposition 3, we can immediately see that if a school accepts a student in some state $\omega$, then the cutoffs are larger in every state where the school is less popular and so accept the student in all those states as well.

THEOREM 4. Consider any market in the continuum economy with correlated preferences model. The equilibrium strategy $\sigma^{*}$ induces a stable matching and, therefore, the deferred acceptance mechanism is stable.

In summary, the results that there is a natural and simple equilibrium, and that this equilibrium produces a stable matching carry over to the case where preferences are correlated, but not perfectly so. Of course, this makes sense as the general logic of the curse of acceptance does not rely on perfect correlation, but requires only some interdependence of student preferences. 


\section{THE WELFARE EFFECTS OF SCHOOL CHOICE}

One of the main motivations for introducing school choice is that it allows parents to provide information on how they rank various schools, and this preference information can then be used by the school district to determine a more efficient assignment than the historical practice of neighborhood assignment. However, if some parents do not know their preferences, less informed parents may be exploited by more informed parents and fall prey to the curse of acceptance. Thus, to the extent that school districts are concerned about the welfare of less informed parents (and they probably are given that the less informed parents may be from economically disadvantaged areas), school choice may actually have significant downsides. We close this paper by showing that the use of secure schools can be seen as a practical compromise: it allows choice for those parents who know their preferences, while still protecting the less informed from being exploited.

We make this point formally in the context of the continuum model from the previous section. We consider student welfare under three alternative school assignment procedures: No Choice (NC), DA with No Secure Schools (DA-NSS), and DA with Secure Schools (DA-SS). DA-SS is simply the procedure in the previous section in which each student is given a secure school and assignments are determined by running the DA algorithm on the submitted preferences. DA-NSS is the same, except students are not given any secure schools and so their priority score at every school is just equal to their priority number. ${ }^{9}$ Under No Choice, students are not asked to submit any information about their preferences and are simply assigned to a school. To be able to make meaningful comparisons, we assume that under No Choice, students are assigned to whatever was their secure school under DA-SS.

We are interested in comparing the welfare for each group of students (informed and uninformed) under each of the procedures. For any agent $j$, let $V_{j}^{\alpha}(\sigma)$ denote agent $j$ 's interim expected utility after she receives her signal about her own preferences, but before the mechanism is run, under school choice procedure $\alpha$ when agents follow strategy profile $\sigma$, where $\alpha=$ SS, NSS, or NC. We say that procedure $\alpha$ (with equilibrium $\sigma$ ) Pareto dominates procedure $\alpha^{\prime}$ (with equilibrium $\sigma^{\prime}$ ) for some set of students $\tilde{J} \subseteq J$ if $V_{j}^{\alpha}(\sigma) \geq V_{j}^{\alpha^{\prime}}\left(\sigma^{\prime}\right)$ for all $j \in \tilde{J}$. Further, if this inequality is strict for a subset $\tilde{\tilde{J}} \subseteq \tilde{J}$ that has strictly positive measure, we say that $\alpha$ strictly Pareto dominates $\alpha^{\prime}$ for $\tilde{J}$.

The first point we make is to formalize the idea that school choice can have a significant downside for uninformed students. In particular, if there are no secure schools, then the uninformed students fall prey to the curse of acceptance and are actually be worse off, in a Pareto sense. Before we can state this formally, we need to analyze equilibrium under DA-NSS. In fact, there turns out to be a very natural equilibrium of the DA-NSS game. Let $\hat{\sigma}$ be the strategy profile in which informed students report their true preferences and uninformed students report schools in ascending order of their priority numbers. We then have the following theorem.

\footnotetext{
${ }^{9}$ We note that once preferences have been submitted, the DA-NSS algorithm is equivalent to the DA with the multiple tie-breaking algorithm considered in the continuum model of Abdulkadiroğlu et al. (2015).
} 
Proposition 4. Consider any market in the continuum economy with correlated preferences model with the adjustment to no secure schools. The strategy profile $\hat{\sigma}$ is an equilibrium of the DA-NSS game.

The outline of the proof of this proposition follows two steps similar to the proof that $\sigma^{*}$ is an equilibrium of DA-SS. First, the cutoff result of Proposition 3 still holds because, just as before, an equal number of uninformed students are assigned to each school. Second, given this cutoff structure, it is optimal for the uninformed students to list schools where they have better priority first, because those are the schools they can get when they are more popular and, thus, mitigate the curse of acceptance the most. While there may be other equilibria, we think that this one is very natural and intuitive (because the uninformed students report the schools they are most likely to get into at the top of their preferences), and we use it to establish our welfare results. Our first main result in this section is that the curse of acceptance means that the uninformed students would prefer no choice. In particular, school choice has the exact opposite of its intended effect.

THEOREM 5. Consider any market in the continuum economy with correlated preferences model with the adjustment to no secure schools. No Choice strictly Pareto dominates DANSS (with equilibrium $\hat{\sigma}$ ) for $U$.

However, when there are secure schools, then school choice does have the intended effect. In particular, the uninformed students get their secure school in either case, while informed students can use their information to improve their own assignment.

THеовем 6. Consider any market in the continuum economy with correlated preferences model. DA-SS (with equilibrium $\sigma^{*}$ ) strictly Pareto dominates No Choice for J.

To summarize, in general, we have seen that uninformed students may be exploited by informed students and end up worse off than if there were no school choice at all due to the curse of acceptance. However, we have also provided school districts worried about these issues with a potential design response to mitigate their effects. Giving each student a secure school can be seen as a practical compromise: it allows choice for those students who know their preferences, which increases efficiency, while protecting uninformed students from the unintended consequences of the curse of acceptance. Overall, the final result is a Pareto-improving allocation.

\section{Conclusion}

This paper introduces correlation in student preferences and differentially informed students into school choice. We show that many standard results fail to hold: the popular deferred acceptance mechanism is no longer strategy-proof or stable, and uninformed students are made worse off. While we focus on DA because it is the mechanism that has gained by far the most traction in practical applications, our results are not specific to DA and can be attributed to a general curse of acceptance: informed students 
are more likely to list the good schools highly precisely because they know which ones they are, and so uninformed students expect that any school that accepts them are of low quality. However, we also show that there is the potential to mitigate these effects by casting the problem as one of priority design. When all students have a secure school, positive results are recovered. Secure schools can be seen as a practical compromise that allows choice and market forces to increase efficiency for those who know their preferences, while protecting uninformed students from the unintended consequences of the curse of acceptance, resulting in a Pareto-improving final allocation.

While our model is stylized, our goal is to point out important issues regarding preference modeling that have not been studied in the school choice literature, and to highlight how they underscore the need to think carefully about the interplay between preference information and the design of priorities. We think our paper opens up many interesting avenues for future research, in particular regarding how priorities should be designed in light of the asymmetric information problem. For instance, one possibility is to make everyone's secure school his neighborhood school, but if less informed and more disadvantaged students are more likely to live closer to lower quality schools, then this may not be a desirable policy. Additionally, the definition need not be "binary," i.e., distributing priorities in such a way that students have a relatively high, though not necessarily guaranteeable, priority number at some school still mitigates, though perhaps not completely eliminates, the negative welfare effects caused by the curse of acceptance. These are important issues to understand to bring these ideas into practice.

One intriguing proposal is the priority redesign undertaken by Boston (discussed in Section 4), in which schools are divided into quality tiers, which are then used to determine each student's "menu." If a school is not on a student's menu, then she is not allowed to list it, which is effectively a way to at least partially distribute priorities based on some objective quality measures. Of course, not all students may place equal importance on these quality measures relative to other factors, which is why choice is still important; at the same time, in light of our results, distributing priorities more evenly across quality tiers may mitigate the curse of acceptance and raise the welfare of less informed students. While we leave the question of the "optimal" way to design priorities for future work, we think that information asymmetry issues should not be ignored in this debate, since, as our results point out, they are likely to have important consequences for student welfare.

\section{Appendix: Proofs}

We introduce additional notation as needed below and then sometimes use that notation again in later proofs, so if notation seems unfamiliar from the main text, refer to previous proofs in this appendix for definitions.

\section{A.1 Proof of the revelation principle}

Let $\psi$ be a mechanism and let $\sigma^{*}$ be an equilibrium of $\psi$. Consider $\sigma=\left(\left(\sigma_{j}^{t}\right)_{j \in I}\right.$, $\left.\left(\sigma_{j}^{*}\right)_{j \in U}\right)$. Slightly abusing notation, let $\sigma_{j}^{-1}\left(P_{j}\right)$ be any $\omega$ such that $P_{j}(\omega)=P_{j}$ (note 
that measurability means that $\sigma_{j}^{t}(\omega) \neq \sigma_{j}^{t}\left(\omega^{\prime}\right)$ implies $\left.P_{j}(\omega) \neq P_{j}\left(\omega^{\prime}\right)\right)$ and let $\sigma_{j}^{-1}\left(P_{j}\right)$ be any arbitrary state $\omega$ for the uninformed students (note that measurability means that $\sigma_{j}^{*}(\omega)=\sigma_{j}^{*}\left(\omega^{\prime}\right)$ for all states). For additional notation, let $\sigma^{-1}(P)=\left(\sigma_{1}^{-1}\left(P_{1}\right), \ldots\right.$, $\left.\sigma_{N}^{-1}\left(P_{N}\right)\right)$, where $P=\left(P_{1}, \ldots, P_{N}\right)$, and let $\sigma_{-j}^{-1}(P)$ denote the inverses of all students other than $j$. Construct the mechanism $\psi^{\prime}$ by letting $\psi^{\prime}(P)=\psi\left(\sigma^{*}\left(\sigma^{-1}(P)\right)\right)$. We want to show that truthful reporting by the informed and $\sigma^{*}$ by the uninformed is an equilibrium strategy of $\psi^{\prime}$. Denote this report in state $\omega$ by $P(\omega)$. First, $\psi^{\prime}(P(\omega))=\psi\left(\sigma^{*}\left(\sigma^{-1}(P(w))\right)=\psi\left(\sigma^{*}(\omega)\right)\right.$ (the last equality holds even if $\sigma_{j}^{-1}$ is $\omega^{\prime} \neq \omega$, because measurability implies that $\left.\sigma_{j}^{*}\left(\omega^{\prime}\right)=\sigma_{j}^{*}(\omega)\right)$. That is, the report $P(\omega)$ determines the same matching in mechanism $\psi^{\prime}$ as $\sigma^{*}(\omega)$ determines in mechanism $\psi$. Second, for any report $P_{j}^{\prime}$ by $j, \psi^{\prime}\left(P_{j}^{\prime}, P_{-j}(\omega)\right)=\psi\left(\sigma^{*}\left(\sigma_{j}^{-1}\left(P_{j}^{\prime}\right), \sigma_{-j}^{-1}\left(P_{-j}(\omega)\right)\right)\right)=$ $\psi\left(\sigma_{j}^{*}\left(\sigma_{j}^{-1}\left(P_{j}^{\prime}\right)\right), \sigma_{-j}^{*}(\omega)\right)$, so by deviating to the report $P_{j}^{\prime}, \psi^{\prime}$ determines the same matching as deviating from $\sigma_{j}^{*}(\omega)$ to $\sigma_{j}^{*}\left(\sigma_{j}^{-1}\left(P_{j}^{\prime}\right)\right)$ in the mechanism $\psi$. As $\sigma^{*}$ is an equilibrium of $\psi$, no deviations yield higher expected payoffs and so the deviation to $P_{j}^{\prime}$ does not yield higher expected payoffs.

\section{A.2 Proof of Proposition 2}

Let $\psi$ be a stable and truthful mechanism. Consider Example 2 and suppose $j_{4}$ 's equilibrium report to $\psi$ has $D P_{j_{4}} C$. Then, in state $\omega^{\prime}=A B C D$, by truthfulness, it is the case that $j_{k}$ reports $\omega^{\prime}$ for $k=1,2,3$. By uniqueness of the stable full matching, $j_{1}$ is matched to $A, j_{2}$ is matched to $D, j_{3}$ is matched to $B$, and $j_{4}$ is matched to $C$. In the full information game, the reports $P_{j_{4}}$ from $j_{4}$ and $\omega^{\prime}$ from $j_{k}$ for $k=1,2,3$ are the true preferences of all students because the mechanism is truthful. The resulting matching is not constrained efficient in the full information game because $\mu_{j_{4}} P_{j_{2}} \mu_{j_{2}}$ and $\mu_{j_{2}} P_{j_{4}} \mu_{j_{4}}$ while $\mu_{j_{k}} P_{j_{k}} \mu_{j_{2}}$ and $\mu_{j_{k}} P_{j_{k}} \mu_{j_{4}}$ for both $k=1,3$.

Alternatively, suppose $j_{4}$ 's equilibrium report to $\psi$ has $C P_{j_{4}} D$. Then, in state $\omega^{\prime \prime}=$ $B A D C$, by truthfulness, it is the case that $j_{k}$ reports $\omega^{\prime \prime}$ for $k=1,2,3$. By uniqueness of the stable full matching, $j_{1}$ is matched to $B, j_{2}$ is matched to $A, j_{3}$ is matched to $C$, and $j_{4}$ is matched to $D$. In the full information game, the reports $P_{j_{4}}$ from $j_{4}$ and $\omega^{\prime \prime}$ from $j_{k}$ for $k=1,2,3$ are the true preferences of all students because the mechanism is truthful. The resulting matching is not constrained efficient in the full information game because $\mu_{j_{4}} P_{j_{3}} \mu_{j_{3}}$ and $\mu_{j_{3}} P_{j_{4}} \mu_{j_{4}}$ while $\mu_{j_{k}} P_{j_{k}} \mu_{j_{3}}$ and $\mu_{j_{k}} P_{j_{k}} \mu_{j_{4}}$ for both $k=1,2$. Thus, $\psi$ is not robustly constrained efficient.

\section{A.3 Proof of Theorem 1}

Recall that a state $\omega \in \Omega$ can be identified with an ordinal ranking of the schools. We write a generic state as

$$
\omega=\left(s^{(1)}, s^{(2)}, \ldots, s^{(M)}\right),
$$

where $s^{(k)}$ denotes the $k$ th best school in state $\omega$. Let $\mu$ denote the full matching induced by the deferred acceptance mechanism when the students choose the strategy $\sigma^{*}$. 
Lemma 1. Let $j$ be an uninformed student. Suppose all students $j^{\prime} \neq j$ follow the strategy $\sigma_{j^{\prime}}^{*}(\cdot)$, and let $j$ choose any arbitrary strategy. Consider a state $\omega=\left(s^{(1)}, \ldots, s^{(M)}\right)$ such that under this strategy profile, we have $\boldsymbol{\mu}_{j}^{\omega}=s^{(k)}$. Then, in any other state $\omega^{\prime}=$ $\left(s^{\prime(1)}, \ldots, s^{(M)}\right)$ where $s^{(m)}=s^{(m)}$ for each $m<k$, we have $\boldsymbol{\mu}_{j}^{\omega^{\prime}} \notin\left\{s^{(1)}, \ldots, s^{(k-1)}, s^{\prime(k)}\right\}$.

Proof. Given the proposed strategies, all of the uninformed students other than $j$ apply to a secure school in the first round and are admitted. Thus, it is without loss of generality to consider the submarket that removes these students and their seats. This means that we can consider a market with only one uninformed student, and the rest of the students are informed.

Consider DA for this submarket in state $\omega$. First, we show that in all steps $m<k$, the informed students who have not matched in a previous step apply to $s^{(m)}$ and the top $q_{s_{m}}$ priority of them are admitted (where we abuse notation and let $q_{s_{m}}$ denote the capacity of $s^{(m)}$ in the submarket). Suppose, for contradiction, that this is not true. By construction of the informed students' preferences, this is only possible if there is some step $m$ where the uninformed student $j$ is matched to $s^{(m)}$ at the end of the step. Since $j$ is ultimately matched to $s^{(k)}$, there is some step $m^{\prime}>m$ where $j$ is rejected from $s^{(m)}$. But there are only informed students applying in steps $m+1, \ldots, m^{\prime}$ and they have all been rejected from schools $s^{(1)}, \ldots, s^{(m)}$, so this contradicts that any student will apply to $s^{(m)}$ in step $m^{\prime}$. This also means that all the informed students who are tentatively assigned in steps $m<k$ to $s^{(m)}$ are matched to $s^{(m)}$ at the end of the algorithm (as otherwise it could only be $j$ 's application that rejects them, but then $j$ would not subsequently get rejected following the same argument just given).

Now let $I_{k}$ denote all informed students who are not assigned to $s^{(m)}$ for some $m<k$. These $I_{k}$ students apply to $s^{(k)}$ in step $k$ and the top $q_{s_{k}}-1$ priority of them are matched to $s^{(k)}$ because $j$ ultimately gets matched to $s^{(k)}$ and, given the previous paragraph, none of the informed students in $I \backslash I_{k}$ ever applies to $s^{(k)}$.

Now consider state $\omega^{\prime}$. Given the first paragraph and that informed students rank the first $k-1$ schools identically, steps $m<k$ follow identically those steps when the state is $\omega$. So the same $I_{k}$ informed students are left that could possibly match with $s^{(k)}$. As $j$ matches to $s^{(k)}$ in state $\omega$, we know that $j$ has lower priority than at most $q_{s_{k}}-1$, which means that if $j$ applies to $s^{(k)}$, then $j$ will match to $s^{(k)}$. Therefore, $j$ will never match to a school $j$ ranks lower than $s^{(k)}$.

So what remains to show is that in state $\omega^{\prime}$, if $j$ ranks $s^{(m)}$ for some $m<k$ or $s^{\prime(k)}$ better than $s^{(k)}$, then $j$ is rejected. As the same informed students are matched to $s^{(m)}$ for each $m<k$ in both states, $j$ must be rejected from each $s^{(m)}$ or else they would have matched to it in $\omega$ as well. For $s^{\prime(k)}$, the top $q_{s_{k}^{\prime}}$ students from $I_{k}$ tentatively match to $s^{\prime(k)}$. Suppose, for contradiction, that $j$ matched to $s^{\prime(k)}$ in state $\omega^{\prime}$. Then $j$ has higher priority than one of these students. But the students who get matched to $s^{\prime(k)}$ in state $\omega$ are a subset of $I_{k}$, so $j$ is not rejected from $s^{\prime(k)}$ in $\omega$, contradicting that $j$ is matched to the school they rank worse, $s^{(k)}$. 
Fixing the strategies of the other agents at $\sigma_{j^{\prime}}^{*}$, for any strategy $\sigma_{j}$ for the uninformed student $j$, let

$$
F\left(k \mid \sigma_{j}\right)=\operatorname{Pr}\left(j \text { matches to a school ranked } k \text { th or better } \mid \sigma_{j}\right)
$$

denote the rank distribution of $j$ 's assignment when she uses strategy $\sigma_{j}$. We now prove a theorem from which Theorem 1 is an immediate consequence. Note that $F\left(\cdot \mid \sigma_{j}^{*}\right)$ firstorder stochastically dominating $F\left(\cdot \mid \sigma_{j}\right)$ immediately implies that $\sigma_{j}^{*}$ is optimal for student $j$.

Theorem 7. For any strategy $\sigma_{j}, F\left(\cdot \mid \sigma_{j}^{*}\right)$ first-order stochastically dominates $F\left(\cdot \mid \sigma_{j}\right)$.

Before the proof, note that Theorem 1 follows immediately given that first-order stochastic dominance implies that expected utility (for any utility function that is decreasing in rank) is larger from $\sigma_{j}^{*}$ than $\sigma_{j}$.

Proof of Theorem 7. Suppose not, i.e., suppose there exists some $\sigma_{j}$ and some $k$ such that $F\left(k \mid \sigma_{j}\right)>F\left(k \mid \sigma_{j}^{*}\right)$. Note that if $j$ follows strategy $\sigma_{j}^{*}$, she gets the same school in every state, call it $\bar{s}$. There are $(M-1)$ ! states where $\bar{s}$ is the best school, $(M-1)$ ! states where it is the second-best school, etc., and so it is easy to see that $F\left(k \mid \sigma_{j}^{*}\right)=(M-$ $1) ! k / M$ !. Consider the minimum $k$ such that $F\left(k \mid \sigma_{j}\right)>F\left(k \mid \sigma_{j}^{*}\right)$. For this $k$, it must be that $j$ gets the $k$ th or better ranked school in strictly more than $k(M-1)$ ! states, and because $k$ is the smallest index for which this is true, $j$ must get exactly the $k$ th ranked school in strictly more than $(M-1)$ ! states.

Partition the state space into groups such that each group contains all of the states for which the best $k$ schools are the same; in other words, two states $\omega=\left(s^{(1)}, \ldots, s^{(M)}\right)$ and $\tilde{\omega}=\left(\tilde{s}^{(1)}, \ldots, \tilde{s}^{(M)}\right)$ belong to the same group $G$ if and only if $s^{(\ell)}=\tilde{s}^{(\ell)}$ for all $\ell=1, \ldots, k$. There are $M ! /(M-k)$ ! groups, and each group contains $(M-k)$ ! states. Note that each group $G$ can be uniquely identified by listing its top $k$ schools in order, $\left(s^{(1)}, \ldots, s^{(k)}\right)$.

Suppose that in state $\omega=\left(s^{(1)}, \ldots, s^{(k)}, \ldots, s^{(M)}\right)$, student $j$ matches to school $s^{(k)}$. Let $G_{\tilde{s}}$ be the group such that $\tilde{s}^{(1)}=s^{(1)}, \ldots, \tilde{s}^{(k-1)}=s^{(k-1)}$, but $\tilde{s}^{(k)}=\tilde{s} \neq s^{(k)}$; in other words, $G_{\tilde{s}}$ is the group that has the same first $(k-1)$ best schools as $G$, but replaces $s^{(k)}$ with $\tilde{s}$. There are $M-k$ possible choices for $\tilde{s}$ and, hence, $M-k$ such groups $G_{\tilde{s}}$. Let $\tilde{S}=$ $S \backslash\left\{s^{(1)}, \ldots, s^{(k)}\right\}$ and define $\tilde{G}=\bigcup_{\tilde{s} \in \tilde{S}} G_{\tilde{s}}$. Lemma 1 implies that for all $\omega^{\prime} \in \tilde{G}$, student $j$ ends up with worse than the $k$ th ranked school. Note that $|\tilde{G}|=(M-k) \times(M-k)$ !.

By our hypothesis, $j$ gets the $k$ th best school in strictly more than $(M-1)$ ! states. Every group $G$ contains $(M-k)$ ! different states, which implies that $i$ must get the $k$ th best school in at least $(M-1) ! /(M-k)$ ! different groups. But by the previous paragraph, for each of these groups $G$, there is an associated $\tilde{G}$ such that $j$ gets strictly worse than the $k$ th best school for all $\omega^{\prime} \in \tilde{G}$. Since $|\tilde{G}|=(M-k) \times(M-k)$ ! and there must be at least $(M-1) ! /(M-k)$ ! such $\tilde{G}$ s, that means that there are at least

$$
(M-k) \times(M-k) ! \times \frac{(M-1) !}{(M-k) !}=(M-k) \times(M-1) !
$$


states where $j$ gets worse than the $k$ th ranked school. Since there are $M$ ! total states, this leaves at most

$$
M !-(M-k) \times(M-1) !=k \times(M-1) !
$$

states where $j$ can get the $k$ th ranked or better school. However, this contradicts that $j$ gets the $k$ th ranked or better school in strictly more than $k \times(M-1)$ ! states.

\section{A.4 Proof of Theorem 2}

Choose some uninformed student $j$. As with the proof of Theorem 1, it is without loss of generality to consider a submarket that has removed all of the other uninformed students $j^{\prime} \in U \backslash\{j\}$ together with the seats they take at their secure schools in equilibrium. We consider the set of students is $I \cup\{j\}$.

We start with the following lemma.

Lemma 2. Assume that $j \succ_{s^{\prime}} j^{\prime}$ for some $j^{\prime}$ assigned to school $s^{\prime}$ in state $\omega=\left(s^{(1)}, \ldots\right.$, $\left.s^{(k-1)}, s^{\prime}, s^{(k+1)}, \ldots, s^{(M)}\right)$. Then, for any state $\tilde{\omega}=\left(\tilde{s}^{(1)}, \ldots, \tilde{s}^{(M)}\right)$ such that $\tilde{s}^{(m)}=s^{(m)}$ for all $m<k$, we have $j \succ_{s^{\prime}} j^{\prime \prime}$ for some $j^{\prime \prime}$ assigned to school $s^{\prime}$ in state $\tilde{\omega}$.

In other words, this lemma says that if student $j$ 's proposed rematching is accepted by school $s^{\prime}$ in some state $\omega$, it will also be accepted in any state $\tilde{\omega}$ where $s^{\prime}$ is ranked lower; i.e., if $s^{\prime}$ accepts $j$ when it is "good," it will also accept $j$ when it is "bad."

Proof of Lemma 2. Consider the DA algorithm in state $\omega$. Since all the uninformed students take their secure school, we need to consider only the informed students. Thus, at each step $m$ of the algorithm, there is a set of unmatched students $I_{m}$, and all of these students apply to school $s^{(m)}$. The $q_{s_{m}}$-highest priority students are thus admitted (again, abusing notation to let $q_{s_{m}}$ be the capacity left after the uninformed students whose secure school is $s_{m}$ are matched to it) and since no new students apply to $s^{(m)}$ at any later step of the algorithm, these students are the ones who will ultimately be assigned to school $s^{(m)}$. In state $\omega, s^{(k)}=s^{\prime}$ and so the $q_{s^{\prime}}$-highest priority students from $I_{k}$ are admitted to $s^{\prime}$. By assumption, in this set there is some $j^{\prime}$ such that $j \succ_{s^{\prime}} j^{\prime}$.

Now consider $\tilde{\omega}$. By construction, in state $\tilde{\omega}$, school $s^{\prime}$ is ranked weakly worse than $k$ th: $s^{\prime}=\tilde{s}^{\left(k^{\prime}\right)}$ for some $k^{\prime} \geq k$. Since the schools ranked $m<k$ are the same as in state $\omega$, the set of students who apply to $s^{\prime}$ in state $\tilde{\omega}$ is a subset of $I_{k}$. This means that the lowestranked student admitted to $s^{\prime}$ in state $\tilde{\omega}, j^{\prime \prime}$, is ranked (weakly) worse than $j^{\prime}$ according to $\succ_{s^{\prime}}$, and so $j \succ_{s^{\prime}} j^{\prime} \succsim_{s^{\prime}} j^{\prime \prime}$.

Continuing with the main proof, let $\bar{s}$ be the secure school that student $j$ lists first in equilibrium and that $j$ receives in every state $\omega$. After the matching is implemented, consider $j$ proposing to form a blocking pair with some other school $s^{\prime}$. Suppose there is a state $\omega$ where $j$ has higher priority than some $j^{\prime}$ that is assigned to $s^{\prime}$ and would prefer $s^{\prime}$ to $\bar{s}$. In particular, let the ranking of $s^{\prime}$ in state $\omega$ be $k$ and let the ranking of $\bar{s}$ be $\ell>k$ :

$$
\omega=\left(s^{(1)}, \ldots, s^{(k-1)}, s^{\prime}, s^{(k+1)}, \ldots, s^{(\ell-1)}, \bar{s}, s^{(\ell+1)}, \ldots, s^{(M)}\right) .
$$


By Lemma 2, $j$ also has priority over some $j^{\prime \prime}$ assigned to $s^{\prime}$ in the state

$$
\tilde{\omega}=\left(s^{(1)}, \ldots, s^{(k-1)}, \bar{s}, s^{(k+1)}, \ldots, s^{(\ell-1)}, s^{\prime}, s^{(\ell+1)}, \ldots, s^{(M)}\right) .
$$

So for every state where $s^{\prime}$ accepts $j$ as the $k$ th ranked school and $\bar{s}$ is the $\ell$ th ranked school for $\ell>k$, there is a symmetric state where $s^{\prime}$ accepts $j$ as the $\ell$ th ranked school, while had $j$ stuck with $\bar{s}$, she would have received the $k$ th ranked school. Since each of these states is equally likely, student $j$ is not better off conditional on being admitted to $s^{\prime}$ for these two states. Summing over all state where $j$ can block with $s^{\prime}$, and where $s^{\prime}$ is better than $\bar{s}$ and the companion states where the rankings are reversed, the total expected utility of rematching with $s^{\prime}$ is less than or equal to staying at $\bar{s}$.

Side note: It is tempting to look at this proof and conclude that every school gives the same expected payoff (conditional on a block being accepted), which is obviously not true. The reason is that the above logic does not apply in reverse: that is, if we start with the fact that $j$ 's block with $s^{\prime}$ is accepted in state $\tilde{\omega}$, we cannot conclude that $j$ 's block will be accepted in state $\omega$. But $j$ is worse off when making the switch in state $\tilde{\omega}$, and if she is not accepted in state $\omega$ to offset this "loss," she will be worse off overall.

\section{A.5 Proof of Proposition 3}

Fix $\omega$. This determines the measure of students of each type who report each ordinal preference relation (following the given strategy $\sigma^{*}$ ). As shown by Abdulkadiroğlu et al. (2015), there is a unique vector of cutoffs $\left(\bar{\ell}_{s_{1}}, \ldots, \bar{\ell}_{s_{M}}\right)$ that can be used to determine the DA assignment. Consider two schools $s_{A}$ and $s_{B}$, and assume without loss of generality (WLOG) that $\operatorname{rank}_{\omega}\left(s_{A}\right)<\operatorname{rank}_{\omega}\left(s_{B}\right)$. For any ordinal preference ranking $P \in \mathcal{P}$, write $P(r)$ for the $r$ th ranked school according to $P$. Partition the ordinal preference space $\mathcal{P}$ into $\mathcal{P}=\mathcal{P}_{A} \cup \mathcal{P}_{B}$, where $P \in \mathcal{P}_{A}$ if and only if $s_{A} P s_{B}$ (and $P \in \mathcal{P}_{B}$ if and only if $s_{B} P s_{A}$ ). For every $P \in \mathcal{P}_{A}$, there is a corresponding $P^{\prime} \in \mathcal{P}_{B}$ with $P(r)=P^{\prime}(t)=s_{A}$ and $P(t)=$ $P^{\prime}(r)=s_{B}$ for some $r<t$, and $P(k)=P^{\prime}(k)$ for all $k \neq r, t$. In words, $P$ and $P^{\prime}$ are exactly the same except that the ranking of schools $s_{A}$ and $s_{B}$ are swapped. Consider some such $P \in \mathcal{P}_{A}$ and corresponding $P^{\prime} \in \mathcal{P}_{B}$. By our assumption on the preference measure, $\lambda_{\omega}(P)>\lambda_{\omega}\left(P^{\prime}\right)>0$.

We want to show that $\bar{\ell}_{s_{A}}<\bar{\ell}_{s_{B}}$. Toward a contradiction, suppose that $\bar{\ell}_{s_{A}} \geq \bar{\ell}_{s_{B}}$. Each uninformed student is matched to his secure school so there is an equal measure $\frac{1}{M}(M-\nu) \lambda_{\omega}(P)$ of uninformed students with preferences $P$ matched to each of $s_{A}$ and $s_{B}$ (because a fraction $1 / M$ have each school as their secure school).

For the informed students, it is helpful to divide them into three distinct classes: (i) students whose secure school is strictly preferred to $s_{A}$; (ii) students whose secure school is $s_{A}$; (iii) students whose secure school is strictly worse than $s_{A}$ (where the rankings

are according to $P$ ). The measure of students in class (i) is $\frac{r-1}{M} \nu \lambda_{\omega}(P)$, the measure of students in class (ii) is $\frac{1}{M} \nu \lambda_{\omega}(P)$, and the measure of students in class (iii) is $\frac{M-r}{M} \nu \lambda_{\omega}(P)$ (recall that $\operatorname{rank}_{P}\left(s_{A}\right)=r$, so there are $r-1$ better schools and $M-r$ worse schools than $s_{A}$ ). 
Now no student in class (i) is matched to $s_{A}$, because $\bar{\ell}_{s_{A}} \geq 1$ and so he/she will get into a school they rank at least as well as $s_{A}$ (in fact, $\bar{\ell}_{s} \geq 1$ for every $s$, as otherwise it could not be full to capacity, and every school is full to capacity because there is an equal measure of seats and students). Students in class (ii) match to $s_{A}$ if and only if their priority scores at the $r-1$ schools they prefer to $s_{A}$ are higher than the cutoffs at (all of) these schools. Thus, the fraction of such students matched to $s_{A}$ is $\prod_{x=1}^{r-1}\left(2-\bar{\ell}_{P(x)}\right)$ (we can assume that $\bar{l}_{s} \leq 2$ for each school as 2 is the largest possible priority score). Students in class (iii) are similar, except that they also must have a low enough priority score at $s_{A}$, and so the total fraction of students in class (iii) matched to $s_{A}$ is $\prod_{x=1}^{r-1}(2-$ $\left.\bar{\ell}_{P(x)}\right) \times\left(\bar{\ell}_{s_{A}}-1\right)$. Combining all of this, the total measure of students with preference $P$ matched to school $s_{A}$ in state $\omega$ is

$$
\frac{\lambda_{\omega}(P)}{M} \times\left[\nu \times \prod_{x=1}^{r-1}\left(2-\bar{\ell}_{P(x)}\right) \times\left(1+(M-r)\left(\bar{\ell}_{s_{A}}-1\right)\right)+(M-\nu)\right] .
$$

We can do an equivalent analysis for the measure of students with preference $P^{\prime}$ who get matched to $s_{A}$ (recalling that under $P^{\prime}$, school $s_{A}$ is ranked $t$ th):

$$
\frac{\lambda_{\omega}\left(P^{\prime}\right)}{M} \times\left[\nu \times \prod_{x=1}^{t-1}\left(2-\bar{\ell}_{P^{\prime}(x)}\right) \times\left(1+(M-t)\left(\bar{\ell}_{S_{A}}-1\right)\right)+(M-\nu)\right] .
$$

Now recall that $P(k)=P^{\prime}(k)$ for all $k<t$, with the exception of $k=r$, where $P(r)=s_{B}$. In particular, we can rewrite (1) as

$$
\frac{\lambda_{\omega}\left(P^{\prime}\right)}{M} \times\left[\nu \times \prod_{x=1, x \neq r}^{t-1}\left(2-\bar{\ell}_{P(x)}\right)\left(2-\bar{\ell}_{s_{B}}\right) \times\left(1+(M-t)\left(\bar{\ell}_{s_{A}}-1\right)\right)+(M-\nu)\right] .
$$

The total measure of students assigned to $s_{A}$ who are of preference type either $P$ or $P^{\prime}$ is

$$
\delta\left(P, s_{A}\right) \lambda_{w}(P)+\delta\left(P^{\prime}, s_{A}\right) \lambda_{\omega}\left(P^{\prime}\right),
$$

where, to simplify the notation, we define $\delta\left(P, s_{A}\right)$ and $\delta\left(P^{\prime}, s_{A}\right)$ as

$$
\begin{aligned}
& \delta\left(P, s_{A}\right)=\frac{1}{M} \times\left[\nu \times \prod_{x=1}^{r-1}\left(2-\bar{\ell}_{P(x)}\right) \times\left(1+(M-r)\left(\bar{\ell}_{S_{A}}-1\right)\right)+(M-\nu)\right] \\
& \delta\left(P^{\prime}, s_{A}\right)=\frac{1}{M} \times\left[\nu \times \prod_{x=1, x \neq r}^{t-1}\left(2-\bar{\ell}_{P(x)}\right)\left(2-\bar{\ell}_{s_{B}}\right) \times\left(1+(M-t)\left(\bar{\ell}_{S_{A}}-1\right)\right)+(M-\nu)\right] .
\end{aligned}
$$

We can do the same analysis for school $s_{B}$. By symmetry, the expressions are the same as above, except $P$ is swapped with $P^{\prime}$ and $s_{A}$ is swapped with $s_{B}$. The total measure of students matched to $s_{B}$ is

$$
\delta\left(P, s_{B}\right) \lambda_{w}(P)+\delta\left(P^{\prime}, s_{B}\right) \lambda_{\omega}\left(P^{\prime}\right)
$$


where the $\delta \mathrm{s}$ in this case are defined as

$$
\begin{aligned}
& \delta\left(P, s_{B}\right)=\frac{1}{M} \times\left[\nu \times \prod_{x=1, x \neq r}^{t-1}\left(2-\bar{\ell}_{P(x)}\right)\left(2-\bar{\ell}_{S_{A}}\right) \times\left(1+(M-t)\left(\bar{\ell}_{s_{B}}-1\right)\right)+(M-\nu)\right], \\
& \delta\left(P^{\prime}, s_{B}\right)=\frac{1}{M} \times\left[\nu \times \prod_{x=1}^{r-1}\left(2-\bar{\ell}_{P(x)}\right) \times\left(1+(M-r)\left(\bar{\ell}_{S_{B}}-1\right)\right)+(M-\nu)\right] .
\end{aligned}
$$

We are now interested in comparing the $\delta$ s. In particular, recall our contradiction hypothesis that $\bar{\ell}_{s_{A}} \geq \bar{\ell}_{s_{B}}$. This immediately implies that $\delta\left(P, s_{A}\right) \geq \delta\left(P^{\prime}, s_{B}\right)$ and $\delta\left(P^{\prime}, s_{A}\right) \geq \delta\left(P, s_{B}\right)$. Adding these two equations and rearranging gives

$$
\delta\left(P, s_{A}\right)-\delta\left(P, s_{B}\right) \geq \delta\left(P^{\prime}, s_{B}\right)-\delta\left(P^{\prime}, s_{A}\right) .
$$

Note also that $\delta\left(P, s_{A}\right)-\delta\left(P, s_{B}\right) \geq 0$ with equality if and only if $\bar{\ell}_{P(x)}=2$ for some $x<r$ (to see this, recall also that $r<t$ ). Further, recall that $\lambda_{\omega}(P)>\lambda_{\omega}\left(P^{\prime}\right)$ by our preference assumption, so we can multiply (2) and obtain the inequality

$$
\left[\delta\left(P, s_{A}\right)-\delta\left(P, s_{B}\right)\right] \lambda_{\omega}(P) \geq\left[\delta\left(P^{\prime}, s_{B}\right)-\delta\left(P^{\prime}, s_{A}\right)\right] \lambda_{\omega}\left(P^{\prime}\right) .
$$

This rearranges to

$$
\delta\left(P, s_{A}\right) \lambda_{\omega}(P)+\delta\left(P^{\prime}, s_{A}\right) \lambda_{\omega}\left(P^{\prime}\right) \geq \delta\left(P^{\prime}, s_{B}\right) \lambda_{\omega}\left(P^{\prime}\right)+\delta\left(P, s_{B}\right) \lambda_{\omega}(P) .
$$

Note what this says: among those students whose ordinal preference types $P_{j} \in\left\{P, P^{\prime}\right\}$, a weakly greater measure are matched to $s_{A}$ than to $s_{B}$. Furthermore, the inequality is an equality if and only if $\bar{\ell}_{P(x)}=2$ for some $x<r$, in which case no students are matched to either $s_{A}$ or $s_{B}$. As $s_{A}$ and $s_{B}$ must be filled to capacity, the inequality must be strict for at least one pair $\left\{P, P^{\prime}\right\}$ in the partition. So if we sum over all of the inequalities for every corresponding pair in the partition, we conclude that in state $\omega$, the total measure of students matched to $s_{A}$ is strictly greater than the total measure of students matched to $s_{B}$. However, this contradicts that the schools have the same measure of informed students in all states. As $s_{A}$ and $s_{B}$ were arbitrary schools such that $\operatorname{rank}_{\omega}\left(s_{A}\right)<\operatorname{rank}_{\omega}\left(s_{B}\right)$, we have established that $\bar{\ell}_{S^{(1)}}<\bar{\ell}_{S^{(2)}}<\cdots<\bar{\ell}_{S^{(M)}}$.

Now we show that $\bar{\ell}_{S^{(1)}}>1$. Suppose, for contradiction, that $\bar{\ell}_{s^{(1)}}=1$ (it cannot be less than 1 , as argued above). Then no student for which $s^{(1)}$ is not his secure school gets matched to $s^{(1)}$. As there is an equal measure of seats and students, all schools must be filled to capacity, so every student whose secure school is $s^{(1)}$ must be matched to $s^{(1)}$. Let $s \neq s^{(1)}$ be any other school. As there are a finite number of schools, information types, and ordinal preferences, for every $\epsilon>0$, there is a set of informed students $\tilde{J} \subset I$ of strictly positive measure such that for all $j \in \tilde{J}$, (i) $j$ 's secure school is $s^{(1)}$, (ii) $s P s^{(1)}$, and (iii) $\ell_{s}(j) \leq \epsilon$. As these students are not matched to $s$, this means that $\bar{\ell}_{s}<1+\epsilon$. As $\epsilon$ can be arbitrarily small, this implies that $\bar{\ell}_{s}=1$. But this means that $\bar{\ell}_{s^{(1)}}=\bar{\ell}_{s}$, which contradicts that all cutoffs are distinct, as we just showed in the previous paragraph.

Finally, we show that $\bar{\ell}_{s^{(M)}}=2$. Suppose, for contradiction, that $\bar{\ell}_{s^{(M)}}<2$. By the above inequalities, we have $\bar{\ell}_{s}<2$ for every school $s$. This means that every school rejects 
students at some step of DA. Let step $k$ be the first step where the last school rejects students. Once a school rejects a student, then the school will be at capacity in every future step and so every school is at capacity at the end of step $k$. That is, students are rejected in step $k$ and every school is tentatively filled to capacity with students at the end of step $k$. This is a contradiction, as there is an equal measure of students and seats.

\section{A.6 Proof of Theorem 3}

Consider an uninformed student $j$. The cardinal utility for a school is some function $v(x)$ of its ordinal rank $x$, where $v$ is strictly decreasing (so that $x=1$ is the best possible rank). Thus, $j$ 's (ex ante) expected utility for school $s$ in state $\omega$ is

$$
\bar{v}_{j}^{\omega}(s)=\sum_{P \in \mathcal{P}} \lambda_{\omega}(P) v\left(\operatorname{rank}_{P}(s)\right) .
$$

For a state $\omega=\left(s^{(1)}, \ldots, s^{(M)}\right)$, we first show that $\bar{v}_{j}^{\omega}\left(s^{(1)}\right)>\bar{v}_{j}^{\omega}\left(s^{(2)}\right)>\cdots>\bar{v}_{j}^{\omega}\left(s^{(M)}\right)$. Consider any positive integers $k<l \leq M$ and, similar to the proof of the previous proposition, consider $P$ and $P^{\prime}$ with $P(r)=P^{\prime}(t)=s^{(k)}$ and $P(t)=P^{\prime}(r)=s^{(l)}$ for some $r<t$, and $P(k)=P^{\prime}(k)$ for all $k \neq r, t$. The contribution to $\bar{v}_{j}^{\omega}\left(s^{(k)}\right)$ from just $P$ and $P^{\prime}$ is $\lambda_{\omega}(P) v(r)+\lambda_{\omega}\left(P^{\prime}\right) v(t)$ and to $\bar{v}_{j}^{\omega}\left(s^{(l)}\right)$ is $\lambda_{\omega}(P) v(t)+\lambda_{\omega}\left(P^{\prime}\right) v(r)$. As $\lambda_{\omega}(P)>\lambda_{\omega}\left(P^{\prime}\right)>0$ (since $P$ is closer to state $\omega$ than $P^{\prime}$ ) and $v(r)>v(t)$ (as $r<t$ ), it follows that the contribution is greater for $\bar{v}_{j}^{\omega}\left(s^{(k)}\right)$ than for $\bar{v}_{j}^{\omega}\left(s^{(l)}\right)$. Partitioning $\mathcal{P}$ into $M ! / 2$ pairs $\left\{P, P^{\prime}\right\}$ and summing over all members of the partition, we conclude $\bar{v}_{j}^{\omega}\left(s^{(k)}\right)>\bar{v}_{j}^{\omega}\left(s^{(l)}\right)$. As $k$ and $l$ were arbitrary, we have established that $\bar{v}_{j}^{\omega}\left(s^{(1)}\right)>\bar{v}_{j}^{\omega}\left(s^{(2)}\right)>\cdots>\bar{v}_{j}^{\omega}\left(s^{(M)}\right)$.

Second, by symmetry, for any other state $\tilde{\omega}=\left(\tilde{s}^{(1)}, \ldots, \tilde{s}^{(M)}\right)$, we have $\bar{v}_{j}^{\tilde{\omega}}\left(\tilde{s}^{(k)}\right)=$ $\bar{v}_{j}^{\omega}\left(s^{(k)}\right)$ for all $k=1, \ldots, M$. With slight abuse of notation, define $\bar{v}_{k}:=\bar{v}_{j}^{\omega}\left(s^{(k)}\right)$ for any $\omega, k$. In other words, we have shown that there are $M$ numbers $\bar{v}_{1}>\cdots>\bar{v}_{M}$ such that, conditional on any state $\omega=\left(s^{(1)}, \ldots, s^{(M)}\right), j$ 's expected utility for school $s^{(1)}$ is $\bar{v}_{1}$, for $s^{(2)}$ is $\bar{v}_{2}$, etc. In other words, her expected utility for a school $s$ depends only on the $\operatorname{rank}_{\omega}(s)$ (note that this may be different from $j$ 's own ordinal ranking of $s$, which she only learns ex post).

Assume that all other players are playing their equilibrium strategy. Since $j$ 's strategy must be measurable with respect to her information, we can identify each of her possible strategies $\sigma_{j}$ with a (mixture over) the space ordinal preference relations $\mathcal{P}$. Let $E U_{j}\left(P_{j}\right)$ be $j$ 's expected utility when she reports $P_{j}$ (and everyone else plays their equilibrium strategy). More formally, using the above definitions,

$$
E U_{j}\left(P_{j}\right)=\sum_{\omega \in \Omega} \sum_{k=1}^{M} \operatorname{Pr}(\omega) \times \operatorname{Pr}\left(j \text { receives } s^{(k)} \mid \sigma_{-j}^{*}, \omega, P_{j}\right) \times \bar{v}_{k} .
$$

In stating the next lemma, we slightly abuse notation and let $\ell_{j}(s)$ denote $j$ 's overall priority score at school $s$ (i.e., $\ell_{j}(s)$ is the priority number for $j$ 's secure school and the priority number plus 1 for all other schools). 
Lemma 3. Let $s_{A}$ and $s_{B}$ be two schools such that $\ell_{j}\left(s_{A}\right)>\ell_{j}\left(s_{B}\right)$, and consider a preference report $P_{j}$ such that $P_{j}(r)=s_{A}$ and $P_{j}(r+1)=s_{B}$. Let $P_{j}^{\prime}$ be the alternative report such that $P_{j}^{\prime}(r)=s_{B}, P_{j}^{\prime}(r+1)=s_{A}$, and, for all other $t \neq r, r+1, P_{j}^{\prime}(t)=P_{j}(t)$. Then $E U_{j}\left(P_{j}\right) \leq E U_{j}\left(P_{j}^{\prime}\right)$.

Given this lemma, consider any arbitrary strategy $P_{j}$ for student $j$ who does not rank her secure school, $\bar{s}$, first, i.e., $P_{j}: s_{1}, s_{2}, \ldots, s_{r-2}, s_{r-1}, \bar{s}, s_{r+1}, \ldots$ By the lemma, $E U_{j}\left(P_{j}\right) \leq E U_{j}\left(P_{j}^{\prime}\right)$, where $P_{j}^{\prime}: s_{1}, s_{2}, \ldots, s_{r-2}, \bar{s}, s_{r-1}, s_{r+1}, \ldots$. Applying the lemma again, $E U_{j}\left(P_{j}^{\prime}\right) \leq E U_{j}\left(P_{j}^{\prime \prime}\right)$, where $P_{j}^{\prime \prime}: s_{1}, s_{2}, \ldots, \bar{s}, s_{r-2}, s_{r-1}, s_{r+1}, \ldots$ Continuing in this manner, we eventually find a strategy $P_{j}^{*}: \bar{s}, s_{1}, s_{2}, \ldots$ such that $E U_{j}\left(P_{j}^{*}\right) \geq E U_{j}\left(P_{j}\right)$. Any strategy that ranks $\bar{s}$ first gives $j$ school $\bar{s}$ in every state, with associated expected utility $\frac{1}{M} \sum_{k=1}^{M} \bar{v}_{k}$. As all strategies that rank $\bar{s}$ first give the same expected utility, any such strategy is optimal for player $j$.

We now prove the lemma.

Proof of Lemma 3. Proposition 3 shows that for any state $\omega=\left(s^{(1)}, \ldots, s^{(M)}\right)$, the lottery cutoffs can be written $\bar{\ell}_{s^{(1)}}<\cdots<\bar{\ell}_{s^{(M)}}$. In addition, note that by the symmetry assumption on preferences, the cutoffs are independent of the state; that is, given any two states $\omega=\left(s^{(1)}, \ldots, s^{(M)}\right)$ and $\tilde{\omega}=\left(\tilde{s}^{(1)}, \ldots, \tilde{s}^{(M)}\right)$, and corresponding vectors of cutoffs, we have $\bar{\ell}_{s^{(m)}}=\bar{\ell}_{\tilde{s}^{(m)}}$ for all $m$. In other words, we can just write $\bar{\ell}_{1}<\cdots<\bar{\ell}_{M}$ for the schools ranked first to last in any state. Let $\boldsymbol{\mu}_{j}^{\omega}\left(P_{j}\right)$ denote $j$ 's match when $j$ reports $P_{j}$ in state $\omega$.

Start by partitioning the state space into $\Omega=\Omega_{A} \cup \Omega_{B}$, where $\omega \in \Omega_{A}$ if and only if $\operatorname{rank}_{\omega}\left(s_{A}\right)<\operatorname{rank}_{\omega}\left(s_{B}\right)$ (and $\Omega_{B}=\Omega \backslash \Omega_{A}$ ). As described in the proof of Proposition 3, for each $\omega_{A} \in \Omega_{A}$, there is a corresponding $\omega_{B}$ that swaps the positions of $s_{A}$ and $s_{B}$, and leaves all other schools the same. Consider one such pair $\left(\omega_{A}, \omega_{B}\right)$.

Let $s=\boldsymbol{\mu}_{j}^{\omega_{A}}\left(P_{j}\right)$ and first suppose that $\operatorname{rank}_{P_{j}}(s)<r$. In other words, $j$ is matched to a school she reported as preferred to $s_{A}$ in state $\omega_{A}$. This implies that $\ell_{j}(s) \leq \bar{\ell}_{\text {rank }_{\omega_{A}}(s)}$ and $\ell_{j}\left(s^{\prime}\right)>\bar{\ell}_{\operatorname{rank}_{\omega_{A}}\left(s^{\prime}\right)}$ for all $s^{\prime}$ with $\operatorname{rank}_{P_{j}}\left(s^{\prime}\right)<\operatorname{rank}_{P_{j}}(s)$. But note that in moving to state $\omega_{B}$, the rankings of $s$ and all such $s^{\prime}$ do not change $\left(s_{B}\right.$ is not one of the $s^{\prime}$ as $\left.\operatorname{rank}_{P_{j}}\left(s_{B}\right)=r+1\right)$, so the cutoffs $\bar{\ell}_{\operatorname{rank}_{\omega_{A}}(s)}$ and $\bar{\ell}_{\operatorname{rank}_{\omega_{A}}\left(s^{\prime}\right)}$ do not change either. Furthermore, by construction, $\operatorname{rank}_{P_{j}}(s)=\operatorname{rank}_{P_{j}^{\prime}}(s)$ and $\operatorname{rank}_{P_{j}}\left(s^{\prime}\right)=\operatorname{rank}_{P_{j}^{\prime}}\left(s^{\prime}\right)$ for all such $s^{\prime}$ and so $j$ is always matched to $s$. In summary, we conclude that $j$ 's match is the same in all of these scenarios: $\boldsymbol{\mu}_{j}^{\omega_{A}}\left(P_{j}\right)=\boldsymbol{\mu}_{j}^{\omega_{B}}\left(P_{j}\right)=\boldsymbol{\mu}_{j}^{\omega_{A}}\left(P_{j}^{\prime}\right)=\boldsymbol{\mu}_{j}^{\omega_{B}}\left(P_{j}^{\prime}\right)=s$.

Second, suppose that $\operatorname{rank}_{P_{j}}(s) \geq r$. For ease of notation, $\operatorname{define}_{\operatorname{rank}_{\omega_{A}}}\left(s_{A}\right)=$ $\operatorname{rank}_{\omega_{B}}\left(s_{B}\right)=k$ and $\operatorname{rank}_{\omega_{A}}\left(s_{B}\right)=\operatorname{rank}_{\omega_{B}}\left(s_{A}\right)=k^{\prime}$, where $k<k^{\prime}$. There are several subcases, depending on the relative magnitudes of $\ell_{j}\left(s_{A}\right), \ell_{j}\left(s_{B}\right), \bar{\ell}_{k}$, and $\bar{\ell}_{k^{\prime}}$. Recall that $\ell_{j}\left(s_{B}\right)<\ell_{j}\left(s_{A}\right)$ (by assumption) and $\bar{\ell}_{k}<\bar{\ell}_{k^{\prime}}$ (by Proposition 3 ), which eliminates many possibilities.

Subcase (i): $\ell_{j}\left(s_{B}\right)<\ell_{j}\left(s_{A}\right) \leq \bar{\ell}_{k}<\bar{\ell}_{k^{\prime}}$. Note that $j$ has a priority score good enough to be admitted to both $s_{A}$ and $s_{B}$ in both states $\omega_{A}$ and $\omega_{B}$. Thus, she will be admitted to whichever school she ranks higher in her preferences, regardless of the state. That is, $\boldsymbol{\mu}_{j}^{\omega_{A}}\left(P_{j}\right)=\boldsymbol{\mu}_{j}^{\omega_{B}}\left(P_{j}\right)=s_{A}$ and $\boldsymbol{\mu}_{j}^{\omega_{A}}\left(P_{j}^{\prime}\right)=\boldsymbol{\mu}_{j}^{\omega_{B}}\left(P_{j}^{\prime}\right)=s_{B}$. 
Subcase (ii): $\ell_{j}\left(s_{B}\right) \leq \bar{\ell}_{k}<\ell_{j}\left(s_{A}\right) \leq \bar{\ell}_{k^{\prime}}$. In this case, if $j$ submits $P_{j}^{\prime}$, then she will be admitted to $s_{B}$ in both states. However, if she submits $P_{j}$, then she will only be admitted to $s_{A}$ in state $\omega_{B}$, since her priority score is not good enough in state $\omega_{A}$. Thus, $\boldsymbol{\mu}_{j}^{\omega_{B}}\left(P_{j}\right)=s_{A}$ and $\boldsymbol{\mu}_{j}^{\omega_{A}}\left(P_{j}\right)=\boldsymbol{\mu}_{j}^{\omega_{A}}\left(P_{j}^{\prime}\right)=\boldsymbol{\mu}_{j}^{\omega_{B}}\left(P_{j}^{\prime}\right)=s_{B}$.

Subcase (iii): $\ell_{j}\left(s_{B}\right) \leq \bar{\ell}_{k}<\bar{\ell}_{k^{\prime}}<\ell_{j}\left(s_{A}\right)$. In this case, $j$ 's priority score is not good enough to be admitted to $s_{A}$ in either state, but is good enough for $s_{B}$ in both states. That is, $\boldsymbol{\mu}_{j}^{\omega_{A}}\left(P_{j}\right)=\boldsymbol{\mu}_{j}^{\omega_{B}}\left(P_{j}\right)=\boldsymbol{\mu}_{j}^{\omega_{A}}\left(P_{j}^{\prime}\right)=\boldsymbol{\mu}_{j}^{\omega_{B}}\left(P_{j}^{\prime}\right)=s_{B}$.

Subcase (iv): $\bar{\ell}_{k}<\ell_{j}\left(s_{B}\right)<\ell_{j}\left(s_{A}\right)<\bar{\ell}_{k^{\prime}}$. In this case, $j$ is matched to the lower-ranked school in both states regardless of which she submits. That is, $\boldsymbol{\mu}_{j}^{\omega_{A}}\left(P_{j}\right)=\boldsymbol{\mu}_{j}^{\omega_{A}}\left(P_{j}^{\prime}\right)=s_{B}$ and $\boldsymbol{\mu}_{j}^{\omega_{B}}\left(P_{j}\right)=\boldsymbol{\mu}_{j}^{\omega_{B}}\left(P_{j}^{\prime}\right)=s_{A}$.

Subcase (v): $\bar{\ell}_{k}<\ell_{j}\left(s_{B}\right) \leq \bar{\ell}_{k^{\prime}}<\ell_{j}\left(s_{A}\right)$. In this case, $j$ 's priority score is not good enough to be admitted to $s_{A}$ in either state, but is good enough to be admitted to $s_{B}$ in state $\omega_{A}$, which happens under both $P_{j}$ and $P_{j}^{\prime}$. In state $\omega_{B}, j$ does not have a good enough priority number for $s_{A}$ or $s_{B}$, and so she gets some school $s$ that is ranked (strictly) worse than $(r+1)$ th. Recall that $P_{j}(t)=P_{j}^{\prime}(t)$ for all $t>r+1$, and so this will be the same school $s$ under both reports in state $\omega_{B}$. To summarize, in this case we have $\boldsymbol{\mu}_{j}^{\omega_{A}}\left(P_{j}\right)=\boldsymbol{\mu}_{j}^{\omega_{A}}\left(P_{j}^{\prime}\right)=s_{B}$ and $\boldsymbol{\mu}_{j}^{\omega_{B}}\left(P_{j}\right)=\boldsymbol{\mu}_{j}^{\omega_{B}}\left(P_{j}^{\prime}\right)=s$ for some $s$ such that $\operatorname{rank}_{P_{j}}(s)=\operatorname{rank}_{P_{j}^{\prime}}(s)=t>r+1$.

Subcase (vi): $\bar{\ell}_{k} \leq \bar{\ell}_{k^{\prime}}<\ell_{j}\left(s_{B}\right)<\ell_{j}\left(s_{A}\right)$. In this case, $j$ does not have a good enough priority score for either $s_{A}$ or $s_{B}$ in either state $\omega_{A}$ or $\omega_{B}$. By similar reasoning to Subcase (v), we have $\boldsymbol{\mu}_{j}^{\omega_{A}}\left(P_{j}\right)=\boldsymbol{\mu}_{j}^{\omega_{A}}\left(P_{j}^{\prime}\right)=\boldsymbol{\mu}_{j}^{\omega_{B}}\left(P_{j}\right)=\boldsymbol{\mu}_{j}^{\omega_{B}}\left(P_{j}^{\prime}\right)=s$ for some $s$ such that $\operatorname{rank}_{P_{j}}(s)=\operatorname{rank}_{P_{j}^{\prime}}(s)=t>r+1$.

Looking back through all of the subcases, $j$ 's assignment is independent of her choice between reporting $P_{j}$ and $P_{j}^{\prime}$ (for a fixed state) in all cases except Subcases (i) and (ii). In Subcase (i), if she reports $P_{j}$, she gets $s_{A}$ in both states. Since both states are equally likely, her expected utility conditional on the true state being $\omega \in\left\{\omega_{A}, \omega_{B}\right\}$ is $\frac{1}{2}\left(\bar{v}_{k}+\bar{v}_{k^{\prime}}\right)$. If she reports $P_{j}^{\prime}$, she gets $s_{B}$ in both states, and again her expected utility conditional on the true state being $\omega \in\left\{\omega_{A}, \omega_{B}\right\}$ is $\frac{1}{2}\left(\bar{v}_{k}+\bar{v}_{k^{\prime}}\right)$. Thus, in this subcase again, $j$ is indifferent between $P_{j}$ and $P_{j}^{\prime}$. Last, consider Subcase (ii). In this case, if she reports $P_{j}$, she receives the $\left(k^{\prime}\right)$ th-ranked school (the worse school of $s_{A}$ and $s_{B}$ ) in both states $\omega_{A}$ and $\omega_{B}$, for a expected utility conditional on $\omega \in\left\{\omega_{A}, \omega_{B}\right\}$ of $\bar{v}_{k^{\prime}}$. If she reports $P_{j}^{\prime}$, she receives $s_{B}$ in both states, for a conditional expected utility of $\frac{1}{2}\left(\bar{v}_{k}+\bar{v}_{k^{\prime}}\right)>\bar{v}_{k^{\prime}}$. In this case, she strictly prefers to report $P_{j}^{\prime}$.

In summary, $j$ always weakly prefers $P_{j}^{\prime}$ to $P_{j}$, and she strictly prefers it if her priority scores fall in Subcase (ii) conditioned on the state being either $\omega_{A}$ or $\omega_{B}$. Formally, $E U_{j}\left(P_{j} \mid \omega \in\left\{\omega_{A}, \omega_{B}\right\}\right) \leq E U_{j}\left(P_{j}^{\prime} \mid \omega \in\left\{\omega_{A}, \omega_{B}\right\}\right)$. As every pair of states is equally likely ex ante, summing over all such pairs gives $E U_{j}\left(P_{j}\right) \leq E U_{j}\left(P_{j}^{\prime}\right)$.

\section{A.7 Proof of Theorem 4}

By the properties of DA, informed students have no justified claims at a school they prefer. Thus, consider an uninformed student $j$. Let her secure school where she has high priority be $\bar{s}$ (and note that she is matched to $\bar{s}$ in the equilibrium). Consider $j$ 
potentially proposing a block with some other school $s^{\prime}$. We use many of the ideas and notation from the Proof of Proposition 3 and Theorem 3. In particular, we again partition the states into two groups $\Omega_{\bar{s}}$ and $\Omega_{s^{\prime}}$ : $\Omega_{\bar{s}}$ consisting of those geoups where the rank of $\bar{s}$ is lower and $\Omega_{s^{\prime}}$ consisting of those groups where the rank of $s^{\prime}$ is lower. Take two states $\omega \in \Omega_{\bar{s}}$ and $\hat{\omega} \in \Omega_{s^{\prime}}$ that differ only in that the relative positions of $\bar{s}$ and $s^{\prime}$ are swapped. Let $\operatorname{rank}_{\omega}\left(s^{\prime}\right)=\operatorname{rank}_{\hat{\omega}}(\bar{s})=k$ and $\operatorname{rank}_{\omega}(\bar{s})=\operatorname{rank}_{\hat{\omega}}\left(s^{\prime}\right)=k^{\prime}$, where $k^{\prime}>k$. Recall that $\bar{\ell}_{k}<\bar{\ell}_{k^{\prime}}$ (Proposition 3) and $\bar{v}_{k}>\bar{v}_{k^{\prime}}$ (proof of Theorem 3). There are three possibilities for the outcome states $\omega$ and $\hat{\omega}$ if $j$ proposes a block with $s^{\prime}$.

Case (i): $\ell_{j}\left(s^{\prime}\right) \leq \bar{\ell}_{k}$. In this case, $i$ will be rematched with $s^{\prime}$ in both states $\omega$ and $\hat{\omega}$. So $i$ 's payoff conditional on the true state being in $\{\omega, \hat{\omega}\}$ is $\frac{1}{2}\left(\bar{v}_{k}+\bar{v}_{k^{\prime}}\right)$ whether she stays with $\bar{s}$ or proposes a block with $s^{\prime}$.

Case (ii): $\bar{\ell}_{k}<\ell_{j}\left(s^{\prime}\right) \leq \bar{\ell}_{k^{\prime}}$. In this case, $i$ is rematched with $s^{\prime}$ in state $\hat{\omega}$, but not in state $\omega$. So $i$ 's payoff conditional on the true state being in $\{\omega, \hat{\omega}\}$ is $\bar{v}_{k^{\prime}}<\frac{1}{2}\left(\bar{v}_{k}+\bar{v}_{k^{\prime}}\right)$. She is worse off from proposing a block.

Case (iii): $\bar{\ell}_{k^{\prime}} \leq \ell_{j}\left(s^{\prime}\right)$. In this case, $i$ will not rematch to $s^{\prime}$ in either state $\omega$ or $\hat{\omega}$ and, hence, she is indifferent between proposing a block or not.

Combining these three cases, we see that conditional on the true state lying in $\{\omega, \hat{\omega}\}, i$ prefers to stay at school $\bar{s}$ (she is indifferent in Cases (i) and (iii), and is strictly better off in Case (ii)). As $j$ is matched to $\bar{s}$ in every state, $j$ does not update students' beliefs over the true state upon observing their own match. That is, she must consider the expected utility of the block conditioned on the state being in $\Omega$. Summing over each of the $M ! / 2$ pairs of states from $\Omega_{\bar{s}}$ and $\Omega_{s^{\prime}}$ does just this. As she (weakly) prefers not proposing the block for each pair, she (weakly) prefers not proposing the block conditioned on the state being in $\Omega$ and, thus, the matching is stable.

\section{A.8 Proof of Proposition 4}

The first thing to show is that if all students choose $\hat{\sigma}$, then Proposition 3 holds as well. Fix a state $\omega$. Let $\gamma_{\omega}(P)$ denote the measure of students who submit preference profile $P$. As the uninformed students are equally likely to have any priority numbers, the measure of uninformed students who submit $P$ is $\frac{M-\nu}{M !}$ for every profile $P$. So $\gamma_{\omega}(P)=\nu \lambda_{\omega}(P)+$ $\frac{M-\nu}{M !}$. In particular, using the same $P$ and $P^{\prime}$ defined in the proof of Proposition 3 , we have $\lambda_{\omega}(P)>\lambda_{\omega}\left(P^{\prime}\right)$ and, therefore, $\gamma_{\omega}(P)>\gamma_{\omega}\left(P^{\prime}\right)$.

The proof follows very similarly to the proof of Proposition 3 and so only the differences are noted here. One conceptual difference though is that we count up the measure of students who submit preference profiles $P$ and $P^{\prime}$ who match to $s_{A}$ and $s_{B}$ rather than the measure of students whose true preferences are $P$ and $P^{\prime}$ who match to $s_{A}$ and $s_{B}$. The measures are actually a little easier to calculate without secure schools. The measure of students assigned to $s_{A}$ who submit preference type $P$ or $P^{\prime}$ is

$$
\delta\left(P, s_{A}\right) \gamma_{\omega}(P)+\delta\left(P^{\prime}, s_{A}\right) \gamma_{\omega}\left(P^{\prime}\right),
$$

where $\delta\left(P, s_{A}\right)$ and $\delta\left(P^{\prime}, s_{A}\right)$ are

$$
\delta\left(P, s_{A}\right)=\prod_{x=1}^{r-1}\left(1-\bar{\ell}_{P(x)}\right) \times \bar{\ell}_{s_{A}},
$$




$$
\delta\left(P^{\prime}, s_{A}\right)=\prod_{x=1, x \neq r}^{t-1}\left(1-\bar{\ell}_{P(x)}\right) \times\left(1-\bar{\ell}_{s_{B}}\right) \times \bar{\ell}_{s_{A}} .
$$

The total measure of students matched to $s_{B}$ is

$$
\delta\left(P, s_{B}\right) \gamma_{\omega}(P)+\delta\left(P^{\prime}, s_{B}\right) \gamma_{\omega}\left(P^{\prime}\right)
$$

where the $\delta s$ in this case are defined as

$$
\begin{aligned}
\delta\left(P, s_{B}\right) & =\prod_{x=1, x \neq r}^{t-1}\left(1-\bar{\ell}_{P(x)}\right) \times\left(1-\bar{\ell}_{s_{A}}\right) \times \bar{\ell}_{s_{B}}, \\
\delta\left(P^{\prime}, s_{B}\right) & =\prod_{x=1}^{r-1}\left(1-\bar{\ell}_{P(x)}\right) \times \bar{\ell}_{s_{B}} .
\end{aligned}
$$

We still have $\delta\left(P, s_{A}\right) \geq \delta\left(P^{\prime}, s_{B}\right)$ and $\delta\left(P^{\prime}, s_{A}\right) \geq \delta\left(P, s_{B}\right)$, and so the rest of the proof follows similarly. The one final difference is that we also may have a weak inequality if $\bar{\ell}_{P(x)}=0$ for all $x=r, \ldots, t-1$, but this is not a problem because without secure schools it is obvious that $\bar{\ell}_{s}>0$ for all $s$ (otherwise, some strictly positive mass of students remains unassigned).

Lemma 3 depends only on the cutoffs increasing and so it applies now as well. In particular, suppose an uninformed student $j$ chose a profile $P_{j}$, where $\operatorname{rank}_{P_{j}}(s)<\operatorname{rank}_{P_{j}}\left(s^{\prime}\right)$ for some $s$ and $s^{\prime}$ where $\ell_{j}(s)>\ell_{j}\left(s^{\prime}\right)$. We cannot directly apply Lemma 3 because $P_{j}$ may not rank them consecutively. However, ranked between $s$ and $s^{\prime}$ there must be consecutively ranked schools where $j$ 's priority is worse at the better ranked school. In particular, there must be some schools $\hat{s}$ and $\hat{s}^{\prime}$ with $\operatorname{rank}_{P_{j}}(\hat{s})=\operatorname{rank}_{P_{j}}\left(\hat{s}^{\prime}\right)-1$ and $\ell_{j}(\hat{s})>\ell_{j}\left(\hat{s}^{\prime}\right)$. For these schools, the lemma does apply to show that $P_{j}^{\prime}: s_{1}, s_{2}, \ldots, \hat{s}^{\prime}, \hat{s}, \ldots$ and $E U_{j}\left(P_{j}\right) \leq E U_{j}\left(P_{j}^{\prime}\right)$. This process can be iterated as long as there exists $s$ and $s^{\prime}$ where $\operatorname{rank}_{P_{j}}(s)<\operatorname{rank}_{P_{j}}\left(s^{\prime}\right)$ and $\ell_{j}(s)>\ell_{j}\left(s^{\prime}\right)$. Eventually this leads to the strategy profile $\hat{\sigma}_{j}$ that ranks schools in increasing order of priority numbers.

\section{A.9 Proof of Theorems 5 and 6}

Theorems 5 and 6 are closely related, and so we prove them jointly. Mathematically, we show the following:

(i) For all $j \in U, V_{j}^{S S}\left(\sigma^{*}\right) \geq V_{j}^{N C} \geq V_{j}^{N S S}(\hat{\sigma})$.

(ii) For all $j \in I, V_{j}^{S S}\left(\sigma^{*}\right) \geq V_{j}^{N C}$.

(iii) There exists a subset $\tilde{J} \subseteq U$ with strictly positive measure such that $V_{j}^{N C}>$ $V_{j}^{N S S}(\hat{\sigma})$ for all $j \in \tilde{J}$.

(iv) There exists a subset $\tilde{J} \subseteq I$ with strictly positive measure such that $V_{j}^{S S}\left(\sigma^{*}\right)>$ $V_{j}^{N C}$ for all $j \in \tilde{J}$. 
To start, consider an uninformed student $j \in U$. Under both DA-SS and NC, $j$ is matched to her secure school in every state, and so $V_{j}^{S S}\left(\sigma^{*}\right)=V_{j}^{N C}=\bar{v}:=\frac{1}{M} \sum_{k=1}^{M} \bar{v}_{k}$. We will show that $V_{j}^{N S S}(\hat{\sigma}) \leq \bar{v}$ for all $j \in U$, which completes the proof of (i).

First, partition the state space $\Omega$ as follows. Take an arbitrary state $\omega$, and suppose that in equilibrium, in state $\omega, j$ is matched to school $s$. Let $r=\operatorname{rank}_{\omega}(s)$. Consider every state $\omega^{\prime}$ that maintains the relative rankings of all schools other than $s$ but moves $s$ to a worse ranking (and includes $\omega$ too for ease of notation later). Formally, define the set

$$
\begin{aligned}
\Omega_{1}= & \left\{\omega^{\prime}: \operatorname{rank}_{\omega^{\prime}}(s) \geq r \text { and } \operatorname{rank}_{\omega}\left(s^{\prime}\right)<\operatorname{rank}_{\omega}\left(s^{\prime \prime}\right)\right. \text { if and only if } \\
& \left.\operatorname{rank}_{\omega^{\prime}}\left(s^{\prime}\right)<\operatorname{rank}_{\omega^{\prime}}\left(s^{\prime \prime}\right) \text { for all } s^{\prime}, s^{\prime \prime} \neq s\right\} .
\end{aligned}
$$

As this just moves $s$ down the rankings, each school other than $s$ (weakly) moves up in rankings and so, by the extension of Proposition 3 in the previous proof, $\bar{\ell}_{s^{\prime}}$ is smaller in state $\omega^{\prime}$ than in $\omega$ for all $s^{\prime} \neq s$. Alternatively, $s$ gets worse and so $\bar{\ell}_{s}$ gets larger. Therefore, as $j$ is matched to $s$ in $\omega, j$ is also matched to $s$ in $\omega^{\prime}$. There is precisely one state in $\Omega_{1}$ where $\operatorname{rank}_{\omega^{\prime}}(s)=k$ for each $k=r, \ldots, M$. Thus, conditional on the true state lying in $\Omega_{1}$, j's expected utility is

$$
\frac{1}{M-r+1} \sum_{k=r}^{M} \bar{v}_{k} \leq \frac{1}{M} \sum_{k=1}^{M} \bar{v}_{k}=\bar{v} .
$$

Now consider any state $\omega \in \Omega \backslash \Omega_{1}$ and form $\Omega_{2}$ in the same way (find the school $j$ is matched to in $\omega$ and then move it down the rankings). By construction, once again, $j$ 's expected utility conditional on the state being in $\Omega_{2}$ is weakly less than $\bar{v}$.

Continue this procedure until we run out of states. Note that it might be that $\Omega_{l} \cap$ $\Omega_{k} \neq \varnothing$ for some $l, k$. However, if this is the case, then either $\Omega_{k} \subset \Omega_{l}$ or $\Omega_{l} \subset \Omega_{k}$, and so we simply choose the larger set to form the partition. To see why set containment holds, suppose $\Omega_{l}$ was created by starting with some $\omega$ where $j$ matches to $s$ and $\operatorname{rank}_{\omega}(s)=$ $r$. If $\Omega_{k} \cap \Omega_{l} \neq \varnothing$, then $\Omega_{k}$ must have been created by starting with some $\omega^{\prime}$ where $j$ matches to $s$ and the rankings of all other schools are preserved. So if $\operatorname{rank}_{\omega^{\prime}}(s)=r^{\prime}<r$, then $\Omega_{l} \subset \Omega_{k}$, and if $\operatorname{rank}_{\omega^{\prime}}(s)=r^{\prime}>r$, then $\Omega_{k} \subset \Omega_{l}$. Now, conditional on each element of the partition, $j$ 's expected utility is at most $\bar{v}$; as all states are equally likely ex ante, $V_{j}^{N S S}(\hat{\sigma}) \leq \bar{v}$. This completes the proof of (i).

To show (ii), consider an informed student $j \in I$ with ordinal preferences $P_{j}$ and let her secure school be $\bar{s}$. Under NC, $j$ is assigned to $\bar{s}$. Under DA-SS, since $j$ knows her preferences and it is optimal for informed students to report truthfully, it is clear that her final match will be (weakly) preferred to $\bar{s}$. Thus, we conclude that $V_{j}^{S S}\left(\sigma^{*}\right) \geq V_{j}^{N C}$.

So far, we have shown that DA-SS weakly Pareto dominates NC for all students, and NC weakly Pareto dominates DA-NSS for the uninformed students. What is left to show is that these Pareto dominance relations are actually strict, which are statements (iii) and (iv) above.

We first show (iv), that a strictly positive mass of students prefer DA-SS to NC. Fix a state $\omega=\left(s^{(1)}, \ldots, s^{(M)}\right)$. Recall that under DA-SS, we have $\bar{\ell}_{s^{(1)}} \in(1,2)$ (Proposition 3). 
Now, for any small $\epsilon>0$, there is a set of informed students of mass $\epsilon^{M} \times \nu$ who have priority $\ell_{j}(s) \leq 1+\epsilon$ for all $s \in S$. By choosing $\epsilon$ such $1+\epsilon \leq \bar{\ell}_{s^{(1)}}$, we have a set of students $I^{\prime}$ who all receive their first-choice school, no matter what it is. Since the distribution of secure schools is independent of preferences, we can find a subset $I^{\prime \prime} \subseteq I^{\prime}$ of strictly positive measure such that for all $j \in I^{\prime \prime}, j^{\prime}$ s first-choice school is different from her secure school. There are many ways to construct this set, but, for example, consider two schools $s, s^{\prime}$ and some ordinal preference $P$ that ranks $s$ first. Then there is a subset $I^{\prime \prime}$ of measure $\epsilon^{M} \times \nu \times \frac{1}{M} \times \lambda_{\omega}(P)$ whose secure school is $s^{\prime}$, but rank school $s$ first and have high enough priority to be admitted to it. All of the students in $I^{\prime \prime}$ strictly prefer DA-SS to NC.

Last, we use a similar argument to show (iii): that a strictly positive mass of uninformed students strictly prefer NC to DA-NSS. To do so, first recall that in every state, the second-to-highest cutoff satisfies $\bar{\ell}_{s^{(M-1)}}<1$. Consider some small $\epsilon>0$ such that $1-\epsilon>\bar{\ell}_{s^{(M-1)}}$. There is a set of uninformed students $\tilde{U} \subset U$ of mass $\epsilon^{M} \times(M-\nu)$ such that for all $j \in \tilde{U}, \ell_{j}(s) \geq 1-\epsilon>\bar{\ell}_{s^{(M-1)}}$ for all schools $s \in S$. In other words, these students will receive the worst school in every state state and, hence, $V_{j}^{N S S}(\hat{\sigma})=0$ for all $j \in \tilde{U}$. Since $V_{j}^{N C}=\bar{v}>0$ for these students, they strictly prefer NC to DA-NSS.

\section{REFERENCES}

Abdulkadiroğlu, Atila, Yeon-Koo Che, and Yosuke Yasuda (2015), "Expanding "choice" in school choice.” American Economic Journal: Microeconomics, 7, 1-42. [1111, 1113, 1115, 1122]

Abdulkadiroğlu, Atila, Parag A. Pathak, and Alvin E. Roth (2005), "The New York city high school match.” American Economic Review Papers and Proceedings, 95, 364-367. [1097]

Abdulkadiroğlu, Atila and Tayfun Sönmez (2003), "School choice: A mechanism design approach." American Economic Review, 93, 729-747. [1095, 1103, 1104]

Antler, Yair (2015), “Two-sided matching with endogenous preferences.” American Economic Journal: Microeconomics, 7, 241-258. [1110]

Ashlagi, Itai and Yannai A. Gonczarowski (2018), "Stable matching mechanisms are not obviously strategy-proof.” Journal of Economic Theory, 177, 405-425. [1109]

Azevedo, Eduardo M. and Jacob D. Leshno (2016), "A supply and demand framework for two-sided matching markets." Journal of Political Economy, 124, 1235-1268. [1111, 1113]

Bernheim, B. Douglas (1984), "Rationalizable strategic behavior." Econometrica, 52, 1007-1028. [1098]

Bikhchandani, Sushil (2017), "Stability with one-sided incomplete information." Journal of Economic Theory, 168, 372-399. [1098, 1108]

Chade, Hector (2006), "Matching with noise and the acceptance curse." Journal of Economic Theory, 129, 81-113. [1098]

Chade, Hector, Gregory Lewis, and Lones Smith (2014), "Student portfolios and the college admissions problem.” Review of Economic Studies, 81, 971-1002. [1098] 
Chakraborty, Archishman, Alessandro Citanna, and Michael Ostrovsky (2010), "Twosided matching with interdependent values." Journal of Economic Theory, 145, 85-105. [1098]

Chakraborty, Archishman, Alessandro Citanna, and Michael Ostrovsky (2015), "Group stability in matching with interdependent values." Review of Economic Design, 19, 3-24. [1098]

Che, Yeon-Koo and Youngwoo Koh (2016), “Decentralized college admissions.” Journal of Political Economy, 124, 1295-1338. [1098]

Doval, Laura (2015), "A theory of stability in dynamic matching markets." Unpublished paper, Department of Economics, Northwestern University. [1109]

Dubins, Lester E. and David A. Freedman (1981), "Machiavelli and the Gale-Shapley algorithm." American Mathematical Monthly, 88, 485-494. [1104]

Dur, Umut M., Scott Duke Kominers, Parag A. Pathak, and Tayfun Sönmez (2018), "Reserve design: Unintended consequences and the demise of Boston's walk zones." Journal of Political Economy, 126, 2457-2479. [1109]

Ehlers, Lars and Aytek Erdil (2010), "Efficient assignment respecting priorities." Journal of Economic Theory, 145, 1269-1282. [1109]

Ehlers, Lars and Jordi Massó (2007), "Incomplete information and singleton cores in matching markets." Journal of Economic Theory, 136, 587-600. [1097]

Ehlers, Lars and Jordi Massó (2015), "Matching markets under (in) complete information.” Journal of Economic Theory, 157, 295-314. [1097]

Ehlers, Lars and Alexander Westkamp (2018), "Strategy-proof tie-breaking in matching with priorities." Theoretical Economics, 13, 1009-1041. [1109]

Ergin, Haluk (2002), "Efficient resource allocation on the basis of priorities." Econometrica, 70, 2489-2498. [1109]

Gale, David and S. Shapley Lloyd (1962), "College admissions and the stability of marriage." American Mathematical Monthly, 69, 9-15. [1104]

Han, Xiang (2018), "Stable and efficient resource allocation under weak priorities." Games and Economic Behavior, 107, 1-20. [1109]

Immorlica, Nicole and Mohammad Mahdian (2005), "Marriage, honesty, and stability." In Proceedings of the 16th ACM-SIAM Symposium on Discrete Algorithms, 53-62. [1112]

Kesten, Onur (2006), "On two competing mechanisms for priority-based allocation problems." Journal of Economic Theory, 127, 155-171. [1109]

Kojima, Fuhito (2013), "Efficient resource allocation under multi-unit demand." Games and Economic Behavior, 82, 1-14. [1109]

Kojima, Fuhito and Parag A. Pathak (2009), "Incentives and stability in large two-sided matching markets.” American Economic Review, 99, 608-627. [1112] 
Kojima, Fuhito, Parag A. Pathak, and Alvin E. Roth (2013), "Matching with couples: Stability and incentives in large markets." Quarterly Journal of Economics, 128, 1585-1632. [1112]

Kumano, Taro (2013), "Strategy-proofness and stability of the Boston mechanism: An almost impossibility result.” Journal of Public Economics, 105, 23-29. [1109]

Lee, Sam-Ho (2009), "Jumping the curse: Early contracting with private information in university admissions." International Economic Review, 50, 1-38. [1098]

Li, Shengwu (2017), “Obviously strategy-proof mechanisms." American Economic Review, 107, 3257-3287. [1109]

Liu, Qingmin (2019), "Rational expectations, stable beliefs, and stable matching." Unpublished paper, Department of Economics, Columbia University. [1098, 1102]

Liu, Qingmin, George J. Mailath, Andrew Postlewaite, and Larry Samuelson (2014), "Stable matching with incomplete information." Econometrica, 82, 541-587. [1098]

McVitie, David G. and Leslie B. Wilson (1971), "The stable marriage problem.” Communications of the ACM, 14, 486-490. [1104]

Miralles, Antonio (2009), "School choice: The case for the Boston mechanism.” In Auctions, Market Mechanisms and Their Applications, volume 14 of Lecture Notes of the Institute for Computer Sciences, Social Informatics and Telecommunications Engineering, 58-60, Springer, Berlin, Heidelberg. [1111, 1113]

Pathak, Parag A. and Peng Shi (2017), How Well Do Structural Demand Models Work? Counterfactual Predictions in School Choice. NBER Working Paper No. 24017. [1109]

Pathak, Parag A. and Tayfun Sönmez (2008), "Leveling the playing field: Sincere and sophisticated players in the Boston mechanism.” American Economic Review, 98, 16361652. [1106]

Pearce, David G. (1984), "Rationalizable strategic behavior and the problem of perfection.” Econometrica, 52, 1029-1050. [1098]

Roth, Alvin E. (1989), “Two-sided matching with incomplete information about others' preferences.” Games and Economic Behavior, 1, 191-209. [1097]

Troyan, Peter (2019), "Obviously strategy-proof implemenation of top trading cycles." International Economic Review, 60, 1249-1261. [1109]

Co-editor Ran Spiegler handled this manuscript.

Manuscript received 2 February, 2019; final version accepted 22 December, 2019; available online 14 January, 2020. 